\title{
Hsa_circ_0058124 promotes papillary thyroid cancer tumorigenesis and invasiveness through the NOTCH3/ GATAD2A axis
}

Yao Yao ${ }^{1,2 \dagger}$, Xinyuan Chen ${ }^{1,2 \dagger}$, Hong Yang ${ }^{2,3 \dagger}{ }^{\text {, Wei Chen }}{ }^{1}$, Yichun Qian ${ }^{1}$, Zhongyi Yan ${ }^{2,4,5}$, Tian Liao ${ }^{6,7}$, Weiping Yao ${ }^{1}$, Wenlan $\mathrm{Wu}^{1}$, Tonghua $\mathrm{Yu}^{1}$, Yun Chen ${ }^{2,3^{*}}$ and Yuan Zhang ${ }^{1 *}$ (D)

\begin{abstract}
Background: Despite a good and overall prognosis, papillary thyroid cancer (PTC) can still affect the quality of life of many patients, and can even be life-threatening due to its invasiveness and metastasis. Emerging studies demonstrate that circular RNAs (circRNAs) participate in the regulation of various cancers. However, the circRNA profile in invasive PTC is still not well understood.

Methods: Competing endogenous RNA (ceRNA) microarrays were performed to determine circRNAs contributed to the tumorigenesis and invasiveness of PTC. Bioinformatics methods were used to narrow down the candidate circRNAs. Quantitative real-time polymerase chain reaction (qRT-PCR) assays revealed a significant upregulation of hsa_circ_0058124 in PTC tissue and a close correlation with a poor prognosis for PTC patients. RNA fluorescence in situ hybridization and Cell fractionation assay were used to investigate the subcellular location of hsa_circ_0058124. Then, we examined the functions of hsa_circ_0058124 in PTC by cell proliferation, cell cycle, apoptosis, migration and invasion assay. Mechanistically, RNA sequencing and GSEA analysis were applied to predict the downstream pathway of hsa_circ_0058124. Dual-luciferase report assays were used to explore the potential miRNA sponge role of hsa_circ_0058124. Western blotting, cell proliferation, cell cycle, cell apoptosis, migration and invasion, and mouse xenograft assay were used to validate the effects of hsa_circ_0058124/NOTCH3/GATAD2A axis on PTC progression.
\end{abstract}

Results: In the current study, a novel hsa_circ_0058124 on 2q35 was identified and explored in PTC. Hsa_circ_0058124 is associated with the malignant features and poor outcomes of PTC patients. Hsa_circ_0058124 acts as an oncogenic driver that promotes PTC cell proliferation, tumorigenicity, tumor invasion, and metastasis, which functions as a competing endogenous RNA to modulate miRNA-218-5p and its target gene NUMB expression, and consequently with repression of the NOTCH3/GATAD2A signaling axis in vitro and in vivo.

Conclusions: This study unveils a novel biomarker panel consisting of the hsa_circ_0058124/NOTCH3/GATAD2A axis which is critical for PTC tumorigenesis and invasiveness and may represent a novel therapeutic target for intervening in PTC progression.

Keywords: Papillary thyroid cancer, Circular RNA, Hsa_circ_0058124, NOTCH3, GATAD2A

\footnotetext{
*Correspondence: yuan_zhang63@163.com; chenyun@njmu.edu.cn

${ }^{\dagger}$ Yao Yao, Xinyuan Chen and Hong Yang contributed equally to this work

${ }^{2}$ Department of Immunology, Key Laboratory of Immune Microenvironment

and Disease, Nanjing Medical University, Nanjing 211166, China

${ }^{1}$ Department of Head and Neck Surgery, Jiangsu Cancer Hospital \& Jiangsu

Institute of Cancer Research \& The Affiliated Cancer Hospital of Nanjing

Medical University, Nanjing 210009, China

Full list of author information is available at the end of the article
}

(c) The Author(s). 2019 Open Access This article is distributed under the terms of the Creative Commons Attribution 4.0 International License (http://creativecommons.org/licenses/by/4.0/), which permits unrestricted use, distribution, and reproduction in any medium, provided you give appropriate credit to the original author(s) and the source, provide a link to the Creative Commons license, and indicate if changes were made. The Creative Commons Public Domain Dedication waiver (http://creativecommons.org/publicdomain/zero/1.0/) applies to the data made available in this article, unless otherwise stated. 


\section{Background}

Thyroid cancer (TC) is the most prevalent endocrine malignancy in the world, and its incidence has ascended steadily over the past decades [1]. It occurs mostly in young women and is the fifth ranking cancer worldwide in women in terms of overall morbidity $[2,3]$. Papillary thyroid cancer (PTC) is the most common histotype of TC, accounting for approximately $85 \%$ of all TC $[1,3,4]$. PTC has a generally good prognosis because of its steady growth rate and retention of iodide uptake. However, despite its overall good prognosis, PTC can still affect the quality of life of many patients and can even become lifethreatening due to invasiveness and metastasis $[5,6]$. Although the overall 5-year survival rate of PTC is $97 \%$, patients with advanced PTC merely have a 5-year survival rate of only 59\% [7]. Extrathyroidal extension (ETE) has long been recognized as an independent predictor of poor outcome in patients with PTC. Not only does such invasive disease result in higher rates of recurrence, but 5-year survival rate is compromised in this patient group [8-10]. This poor clinical prognosis of invasive PTC emphasizes the urgency of exploring the mechanism of invasive PTC and developing novel anticancer agents that could intervene in PTC progression.

Both genetic and environmental factors are crucial contributors to PTC, but the role of etiology is still not well understood [3]. However, many large-scale and multi-tissue sequencing programs employing next-generation sequencing technique and bioinformatics methods have been carried out in recent years. Annotation of the data derived from these experiments revealed that only $2 \%$ of the genes in the entire human genome are protein coding genes, while the majority are noncoding genes [11]. Among the noncoding transcripts, circular RNAs (circRNAs) represent a typical class generated by backsplicing and characterized by covalently closed loop structures that lack either $5^{\prime}$ to $3^{\prime}$ polarity and a polyadenylated tail. Accumulating studies have found that circRNAs are involved in various physiological and pathophysiological processes, for instance, modulating alternative splicing [12], sponging microRNA (miRNA) [13], and regulating protein-RNA interactions and genes expression [14]. CircRNAs have been detected in various eukaryotes and participate in the pathology of disease $[15,16]$. Recent literature has also indicated dysregulated expression of several circRNAs in human cancer [17-19]. Nevertheless, little is known about the relationship between circRNAs and the tumorigenesis and invasiveness of PTC $[20,21]$. Due to their closed structure, circRNAs have a high level of stability and resistance to RNA degradation. Therefore, they are likely to be promising and technically suitable biomarkers for PTC and can possibly function as molecular targets in PTC treatment.
In the present study, we detected the differential expression of hundreds of circRNAs in PTC tissues by competing endogenous RNA (ceRNA) microarrays. We then used a bioinformatics method to screen 13 circRNAs that might contribute to the tumorigenesis and invasiveness of PTC. Quantitative real-time polymerase chain reaction (qRT-PCR) assays revealed a significant upregulation of hsa_circ_0058124 in PTC tissue and a close correlation with a poor prognosis for PTC patients. These findings implied that hsa_circ_0058124 may be a novel oncogene associated with PTC tumorigenesis and invasiveness. We also found that hsa_circ_0058124 affects the proliferation, apoptosis, migration, and invasiveness abilities of PTC cells in vitro and in vivo. Mechanistically, we revealed that hsa_circ_0058124 may exert its biological effects in PTC in part through serving as a competing endogenous RNA to modulate miRNA-218-5p and its target gene NUMB expression, subsequently with repression of NOTCH3/GATAD2A axis. Taken together, the results revealed a novel biomarker panel consisting of the hsa_circ_0058124/NOTCH3/GATAD2A axis that is critical in PTC tumorigenesis and invasiveness and may be a novel therapeutic target to intervene PTC progression.

\section{Materials and methods}

\section{Ethics statement and patient tissue samples}

This study was approved by the Ethics Committee of Nanjing Medical University and was conducted in accordance with the government policies and the Declaration of Helsinki. PTC and paired adjacent normal tissues were obtained from 92 patients (Nanjing Medical University Affiliated Cancer Hospital, Nanjing) with a definite pathological diagnosis of PTC during operation. The adjacent normal tissues were collected $3 \mathrm{~cm}$ away from the PTC edge and were confirmed no tumor cells by two specialized pathologists. All samples were immediately frozen in liquid nitrogen after collection. After histopathological vetting, a total of 12 samples, consisting of eight PTC samples (including four invasive tumor tissues with extrathyroidal extension and metastasis and four non-invasive tumor tissues with no extrathyroidal extension or metastasis) and four matching adjacent normal tissues, were ultimately selected for ceRNA microarray analysis.

\section{RNA isolation and microarrays}

The microarray experiments were performed by Shanghai Biotechnology Corporation (SBC) following the protocol of Agilent Technologies Inc. (CA, USA). Briefly, total RNA was isolated and purified using a mirVana $^{\text {Th }}$ miRNA Isolation Kit (Ambion, TX, USA), according to the manufacturer's instructions. RNA samples from each group were then used to generate labeled complementary RNA (cRNA) targets using the Low Input Quick Amp WT Labeling Kit (Agilent 
Technologies, CA, USA) for the SBC human ceRNA microarray $(4 \times 180 \mathrm{~K})$. The labeled cRNA targets were then hybridized with the slides and the slides were scanned on the Agilent Microarray Scanner (Agilent Technologies, CA, USA). Data were extracted with Feature Extraction software 10.7 (Agilent Technologies, CA, USA). CircRNAs with high expression abundances, expression levels with more than 2fold alteration, and a $P<0.01$ were selected for further analysis.

\section{RNA preparation and quantitative real-time PCR (qRT-PCR)}

Total RNA extraction and quantification, RNA purification, and cDNA synthesis were conducted as described previously [22]. For RNase R treatment, $2 \mu$ g total RNA was incubated for $15 \mathrm{~min}$ at $37^{\circ} \mathrm{C}$ with or without $3 \mathrm{U} /$ $\mu \mathrm{g}$ RNase R (Epicentre Technologies, WI, USA). To detect RNA expression, quantitative real-time PCR (qRTPCR) was performed with PowerUp ${ }^{\mathrm{m}} \mathrm{SYBR}^{\mathrm{m}}$ Green Master Mix (Thermo Fisher, MA, USA) and the Applied Biosystems StepOnePlus ${ }^{\mathrm{Tn}}$ Real-time PCR Detection System (Life Technologies, CA, USA). The relative gene expression was calculated using the $2^{-\Delta C T}$ method normalized to GAPDH, and the fold change of gene expression was calculated by the $2^{-\Delta \Delta C T}$ method. The primer sequences are shown in Additional file 1: Table S1. Bulge-loop $^{\text {tw }}$ miRNA qRT-PCR Primer Sets (one RT primer and a pair of qPCR primers for each set) specific for miR-218-5p was designed by RiboBio (Guangzhou, China). The relative expression of miR-218-5p was normalized to human U6 snRNA.

\section{Cell culture and transfection}

TPC-1 and K1 cells were purchased from University of Colorado Cancer Center Cell Bank. These PTC cell lines were maintained in a humidified incubator $(95 \%$ air $/ 5 \% \mathrm{CO}_{2}$ ) at $37^{\circ} \mathrm{C}$ in $90 \%$ RPMI 1640 (Invitrogen, CA, USA) supplemented with $10 \%$ fetal bovine serum (FBS; Invitrogen, CA, USA), $100 \mathrm{U} / \mathrm{ml}$ penicillin, and $100 \mu \mathrm{g} / \mathrm{mL}$ streptomycin. The cells were passaged at a $1: 3$ ratio in $10 \mathrm{~cm}$ dishes once $90 \%$ confluence was reached. All PTC cell lines used in this study were reported previously [21, 23]. Purchased siRNAs (RealGene, Nanjing, China) were used in transfection experiments. The TPC-1 and $\mathrm{K} 1$ cells were cultured to $50-60 \%$ confluence, and the transfection was carried out with Lipofectamine 3000 (Invitrogen, CA, USA). Sequences of the siRNAs are shown in Additional file 1: Table S2. To construct a plasmid expressing NUMB, the full-length human NUMB sequence (NCBI Reference Sequence: NM_001005745.1) was synthesized and cloned into the pCDNA3.1 vector (RealGene, Nanjing, China).

\section{Fluorescent in situ hybridization (FISH)}

Hsa_circ_0058124. FISH staining was performed according to the manufacturer's instructions. Briefly, cells were washed with a solution of $0.5 \%$ Triton X-100 in $1 \times$ PBS, an appropriate amount of anti-HNF1A-AS1 oligodeoxy-nucleotide probe (RiboBio, Guangzhou, China) was added in a hybridization solution containing $1 \%$ blocking solution, and hybridization was allowed to occur overnight in a humid chamber at $37^{\circ} \mathrm{C}$. The cells were then given three 5 -min washes in the dark in $4 \times$ sodium citrate buffer (SSC), $2 \times$ SSC, and $1 \times$ SSC containing $0.1 \%$ Tween -20 at $42{ }^{\circ} \mathrm{C}$. The cells were then given one 5-minwash in phosphate buffered saline (PBS) at room temperature and then stained with $4^{\prime}$, 6-diamidino-2-phenylindole (DAPI). The cells were then given three 5 -min washes with $1 \times$ PBS before imaging by confocal laser scanning microscopy (Zeiss LSM 710 confocal microscope, Germany) to detect the location of hsa circ_0058124 in the PTC cells.

\section{Cell fractionation assay}

Cytoplasmic and nuclear RNA was acquired using a Cytoplasmic and Nuclear RNA Purification Kit (Invitrogen, CA, USA). Briefly, the cells were harvested and incubated for $10 \mathrm{~min}$ with lysis solution on ice. The cells were then centrifuged for $3 \mathrm{~min}$ at $12000 \mathrm{~g}$. The supernatant was collected for cytoplasmic RNA and the nuclear pellet was used for nuclear RNA extraction. GAPDH was used as the cytoplasmic endogenous control. The U6 small nuclear RNA was used as the nuclear endogenous control.

\section{Cell viability assay}

Cells were seeded at a density of $2 \times 10^{3}$ cells per well $(100 \mu \mathrm{L})$ in a 96-well plate. Cell viability was evaluated with the cell counting kit-8 (CCK-8; Bimake, Shanghai, China) after $0,24,48,72$, and $96 \mathrm{~h}$ of transfection. The absorbance was determined with a BioTek Synergy HTX multi-mode reader at $450 \mathrm{~nm}$.

\section{Colony formation assay}

Crystal violet (KeyGen, China) was used to detect colony formation. About 200 cells were seeded in each well of 6-well plates. After transfection, the growth medium was changed every 3 days. After 9 days, the cells were fixed with $75 \%$ ethanol for $2 \mathrm{~h}$, followed by staining with $0.2 \%$ crystal violet for $2 \mathrm{~h}$, and colonies ( $>50$ cells/colony) were counted and photographed. Each experiment was performed three times independently.

\section{5-ethynyl-2'-deoxyuridine (EdU) assay}

EdU assays were performed using the Cell Light ${ }^{\mathrm{tm}}$ EdU kit (RiboBio, Guangzhou, China). Briefly, $6 \times 10^{4}$ cells were seeded in well apiece of a 24-well plate. After transfection for $48 \mathrm{~h}$, the culture medium was supplemented with 
$50 \mu \mathrm{M}$ EdU for another $2 \mathrm{~h}$. The cells were fixed with $4 \%$ formaldehyde for $30 \mathrm{~min}$ and incubated with glycine (2 $\mathrm{mg} / \mathrm{mL}$ ) for $10 \mathrm{~min}$. The cells were then permeabilized with $0.5 \%$ Triton X-100 for $20 \mathrm{~min}$, and then Hoechst 33342 were added. Cell proliferation (the percentage of EdU positive cells) was then determined by fluorescence microscopy (Olympus IX73, Japan). Assays were performed independently three times in triplicate.

\section{Cell cycle analysis and apoptosis assay}

After transfection for $48 \mathrm{~h}$, the cells were collected for cell cycle and apoptosis assays. The apoptosis assay was performed with the Apoptosis Detection Kit (BD Pharmingen, CA, USA) by suspending the cells in $100 \mu \mathrm{L}$ binding buffer containing $5 \mu \mathrm{L} \mathrm{PE}$ and $5 \mu \mathrm{L}$ 7-ADD, incubating them in the dark for $15 \mathrm{~min}$, and adding $400 \mu \mathrm{L}$ binding buffer to resuspend the cells. The cells were divided into viable cells, dead cells, early apoptotic cells, and late apoptotic cells. The early and late apoptotic cells were measured. For cell cycle analysis, the cells were fixed in $75 \%$ ethanol overnight at $-20^{\circ} \mathrm{C}$, then stained with propidium iodide (PI) for 30 min, away from light. The cells were analyzed by flow cytometry (FACS Calibur Flow Cytometer, BD Biosciences, USA) to quantify the cell cycle or cell apoptosis. All experiments were performed independently in triplicate.

\section{Cell migration and invasion assay}

Transwell migration and invasion abilities were assessed using $8 \mu \mathrm{m}$ pore transwell chambers either without Matrigel (for migration assays) or with Matrigel (for invasion assays). Each well of the upper chamber was filled with $300 \mu \mathrm{L}$ of serum-free RPMI 1640 medium containing $1 \times 10^{5} / \mathrm{mL}$ infected cells. The lower chambers contained $700 \mu \mathrm{L}$ complete medium. The cells were fixed in $4 \%$ paraformaldehyde and stained with crystal violet after a $48-\mathrm{h}$ incubation. The cells remaining in the top chamber were removed with cotton swabs, and imaged by light microscopy (Olympus, China). All assays were performed three times independently using triplicate wells.

\section{Scratch wound assay}

Scratch wound assays were conducted by measuring the widths of the scratches at different times. Each well of 6well plates was plated with $3 \times 10^{5}$ cells. After $48 \mathrm{~h}$ incubation, when the well was completely covered with cells, a straight scratch was made with a $200 \mu$ pipette tip. Images of the same fields were taken at different time points $(0,12,24,30,36$, and $40 \mathrm{~h})$ after the scratch. Images at different time points were used to create an animation sequence in accordance with the timeline. The experiment was repeated at least three times independently.

\section{RNA sequencing}

We transiently transfected $3 \times 10^{6}$ TPC- 1 cells with 100 $\mathrm{nM}$ indicated siRNAs for $48 \mathrm{~h}$, and the total RNA samples were collected using TRIzol reagent for whole-genome transcriptome profiling by RNA sequencing. The RNA sequencing and bioinformatics data analysis were performed by Shanghai Biotechnology Corporation. In brief, total RNA was extracted using the RNeasy Micro Kit (Qiagen, Hilden, Germany) and purified with the RNAClean XP Kit (Beckman Coulter, Inc., CA, USA). Paired-end libraries were then synthesized using the TruSeq ${ }^{\circ}$ RNA Sample Preparation Kit (Illumina, USA) following the TruSeq ${ }^{\circ}$ RNA Sample Preparation Guide. The purified libraries were sequenced on the Illumina HiSeq X-ten (Illumina, USA). For data processing, we used Hisat2 (version:2.0.4) [24] to map the cleaned reads to the human GRCh38 reference genome with two mismatches. After genome mapping, Stringtie (version:1.3.0) [25] was run with a reference annotation to generate FPKM (Fragments Per Kilobase of transcript per Million mapped reads) values for known gene models. The $P$-value and FDR were used to define the threshold of significance.

\section{KEGG analysis and gene set enrichment analysis (GSEA)}

To gain insight into hsa_circ_0058124-mediated molecular pathways in PTC, we used the Kyoto Encyclopedia of Genes and Genomes (KEGG) pathway analysis (https://www.kegg.jp). GSEA was also performed using the Broad Institute GSEA version 3.0 software. The gene sets used for the enrichment analysis were downloaded from the Molecular Signatures Database (MsigDB, http://software.broadinstitute.org/gsea/index.jsp).

\section{Western blotting}

The proteins in PTC cells were extracted using the Total Protein Extraction Kit (Thermo Fisher, MA, USA). The protein extracts $(30-40 \mu \mathrm{g})$ were separated on $12 \%$ sodium dodecyl sulfate polyacrylamide gel electrophoresis gels and then electrophoretically transferred to polyvinylidene difluoride membranes (Millipore, USA). After blocking in $5 \%$ non-fat milk for $2 \mathrm{~h}$, the membranes were incubated overnight at $4{ }^{\circ} \mathrm{C}$ with primary antibodies recognizing N3ICD, HES1 (1:1000 dilution; Cell Signaling Technology, USA), NUMB (1:1000 dilution; Abcam, UK), GATAD2A (1:1000; Proteintech, USA), and GAPDH (1:1000 dilution; Proteintech, USA). After incubation with secondary antibodies (1:5000 dilution; Jackson ImmunoResearch, PA, USA), the protein bands were visualized by chemiluminescence using a GE Amersham Imager 600 (GE, USA).

\section{Dual-luciferase reporter assay}

To construct luciferase reporter vectors, the full length of hsa_circ_0058124 or the 3'UTR fragment of NUMB 
(NM_001005745.1; 3'UTR: 2104-3467) containing the predicted potential binding sites were cloned at the XhoI and NotI sites into the pmiR-RB-REPORT ${ }^{\text {tw }}$ luciferase reporter vector (RiboBio., Guangzhou, China). The hsa circ_0058124 mutant sequence or the 3'UTR mutant sequence of NUMB, in which seven nucleotides were mutated within the miR-218-5p binding sites, were also constructed into the vector.

For luciferase activity assay, each construct was cotransfected with indicated miRNAs (RiboBio) in $293 \mathrm{~T}$ cells using Lipofectamine 3000 (Invitrogen) for $48 \mathrm{~h}$. Luciferase assays were performed with the Dual-Luciferase Reporter Assay System (Promega, WI, USA) according to the manufacturer's instructions. Luminescent signal was quantified by BioTek Synergy HTX multi-mode reader, and luciferase activity was presented by relative hRluc/ hluc ratio.

\section{Immunohistochemistry (IHC) analysis}

All tissues were fixed overnight in a formalin solution, dehydrated in ethanol, embedded in paraffin, and sectioned at $5 \mu \mathrm{m}$. Then, the slides were treated with xylene and ethanol to remove the paraffin. The slides were blocked with 5\% normal goat serum and incubated with anti-GATAD2A overnight at $4{ }^{\circ} \mathrm{C}$. After washing with PBS, the slides were incubated with goat anti-rabbit horseradish peroxidase (Jackson ImmunoResearch, PA, USA) for $1 \mathrm{~h}$ at room temperature, and was detected the immunohistochemical reactions using a DAB kit (Vector Laboratories, CA, USA). The slides were examined under a phase contrast light microscope (Olympus IX73, Japan), and the average integral optical density of each positively stained slide was measured using an Image-Pro plus 6.0 image analysis system. Three areas were chosen randomly from each section for measurement.

For validation of differentially expressed genes, the IHC data of thyroid cancer tissues and normal thyroid tissues were downloaded from Human Protein Atlas [26] available from www.proteinatlas.org. Protein expression levels were scaled based on an overall antibody staining score ranging from not detected to high.

\section{Animal studies}

All animal experiments were performed in accordance with a protocol approved by the Institutional Animal Care and Use Committee of Nanjing Medical University. Nude mice (5 in each group) aged 5-6 weeks were injected subcutaneously with $0.1 \mathrm{ml}$ of a cell suspension containing $3.0 \times 10^{6}$ TPC-1 cells for the control group, the LV-sh-hsa_circ 0058124 group, the LV-sh-NOTCH3 group, the LV-shGATAD2A group, the LV-sh-hsa_circ_0058124 together with LV-sh-NOTCH3 group or the LV-sh-hsa_circ 0058124 together with LV-sh-GATAD2A group (Genechem, Shanghai, China) in the right flank.
After the tumor was palpable, it was measured every 3 days. Its volume was calculated according to the formula (volume $=$ length $\times$ width $^{2} \times 0.5$ ).

\section{Statistical analysis}

Data analysis was handled with SPSS ver. 19.0 (SPSS, Chicago, IL, USA). All experimental data were expressed as the mean \pm S.E.M. For continuous variables were analyzed with a two-sided independent $\mathrm{t}$-test or paired $\mathrm{t}$-test when data assumed to have an equal variance per test; otherwise, the Wilcoxon signed-rank test was used to perform the comparisons. Pearson's chi-squared test was used for comparisons of categorical variables. In this study we considered $P<0.05$ as statistically significant.

\section{Results}

CircRNA and mRNA profiling in human PTC tissues and hsa_circ_0058124 characterization

A total of twelve fresh PTC tissue samples, decribed in the previous paragraph, were selected for ceRNA microarray analysis. Detailed information of patient characteristics is provided in Additional file 1: Table S3.

The circRNA expression in the PTC samples was profiled using a $4 \times 180 \mathrm{~K}$ ceRNA microarray (circRNA, lncRNA, and mRNA). The differentially expressed circRNAs were identified by fold-change filtering (|fold change $\mid>2)$ and the Student's t-test $(P$-value $<0.01)$, which revealed 607 circRNAs that were significantly differentially expressed in the invasive tumor vs. adjacent normal tissue set (Fig. 1a) and 49 circRNAs that were significantly differentially expressed in invasive vs. noninvasive tumor set (Fig. 1b). Hierarchical clustering was then performed to demonstrate the circRNA expression patterns among the sets (Fig. 1a, b).

To investigate the contribution of circRNAs to the tumorigenesis and invasiveness of PTC, we calculated the intersection of differential expressions of circRNA between the invasive vs. non-invasive tumor set and the invasive tumor vs. adjacent normal tissue set. This narrowed the number of candidate circRNAs down to 13 . Figure 1c and $\mathrm{d}$ showed the Venn diagrams for this intersection and identified 11 significantly upregulated circRNAs and 2 downregulated circRNAs that overlapped the results of the previous comparisons (Additional file 1: Table S4). The mRNA expression profiles among the sets were also demonstrated using hierarchical clustering analysis and Kyoto Encyclopedia of Genes and Genomes (KEGG) pathway enrichment analysis (Fig. 2a, b). To investigate the potential target gene of the identified 13 circRNAs, a coexpression network analysis was used to screen these circRNAs in the eight PTC tumor samples (Fig. 2c). We also randomly selected 6 target genes to validate this circRNA-mRNA coexpression by qRT-PCR in 20 PTC tissues (Additional file 2: Figure S1). We then 


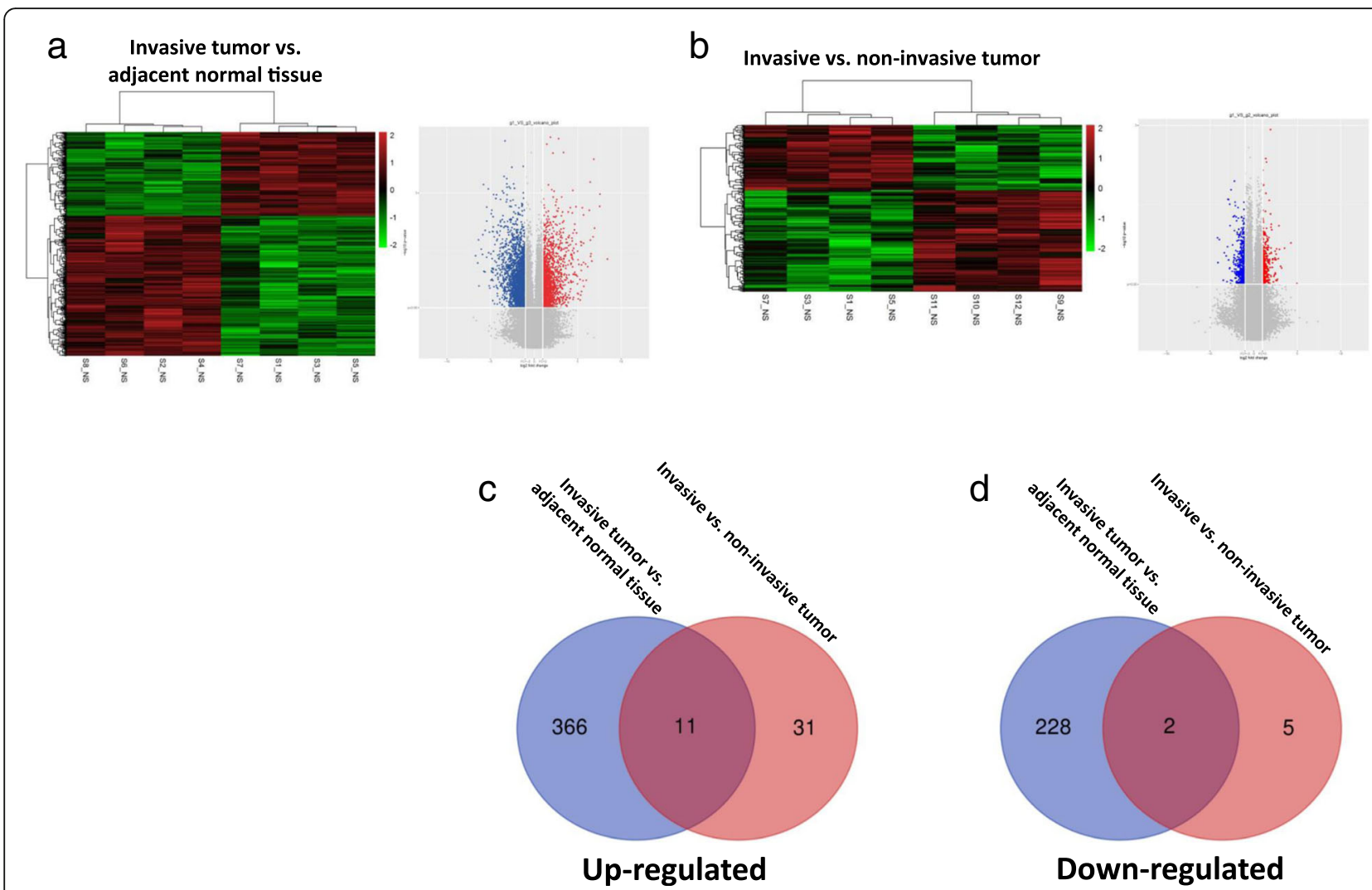

Fig. 1 Screening of PTC-related circRNAs using multiple microarrays and bioinformatics. Hierarchical clustering analysis and volcano plots of significantly differentially expressed circRNAs in the invasive tumor vs. adjacent normal tissue set (a) and the invasive vs. non-invasive tumor set (b). c, d The Venn diagrams demonstrated the significantly changed circRNAs that overlapped in the foregoing comparison in PTC

experimentally validated these 13 circRNA expression levels by qRT-PCR in larger-scale PTC tissue samples. The qRTPCR results indicated hsa_circ_0058124 showed highest fold-change in the PTC tissues than in the control tissues, and was significantly upregulated in the invasive PTC tissues compared with non-invasive tumor tissues (Additional file 2: Figure S2). We therefore chose hsa_circ_ 0058124 for further analysis. The coexpression network for hsa_circ_0058124 was also shown in Fig. 2d.

Hsa_circ_0058124 is located on chromosome 2, is 864 base pairs (bp) in length, and consists of 5 exons (exons 15-19) from its host gene Fibronectin 1 (FN1) genome (Fig. 3a; Additional file 3). FN1, a fundamental component of the extracellular matrix, has been considered as a biomarker of PTC, and an important modulator of thyroid cancer aggressiveness [10, 27]. We avoided trans-splicing or genomic rearrangements, including head-to-tail splicing, by employing several universal circRNA detection methods [28]. We first designed convergent primers to amplify FN1 mRNA (a 184-bp fragment) and divergent primers to amplify hsa_circ_0058124 (a 83-bp fragment). We used cDNA and genomic DNA from two randomly selected PTC tissue and two PTC cell lines (TPC-1 and K1) as templates to amplify hsa_circ_0058124 from cDNA using only divergent primers (an expected 83-bp fragment), while no amplification product was observed from genomic DNA (Fig. 3b). We also used Sanger sequencing to confirm the head-to-tail splicing in the RT-PCR product of hsa_circ_0058124 with the expected size (Fig. 3c). RNase $\mathrm{R}$ is an exoribonuclease that can degrade RNA from its $3^{\prime}$ to $5^{\prime}$ end but does not act on circRNA [29]. In contrast to the linear FN1 mRNA, hsa_circ 0058124 was resistant to RNase R (Fig. 3d). The cell fraction assay and FISH analysis against hsa_circ 0058124 showed that hsa_circ_0058124 was primarily located in the nucleus and also existed in cytoplasm (Fig. 3e-f). Our results implied that hsa_circ_0058124 is a bona fide exonic circRNA that is abundant and stable in TPC-1 cells.

\section{Upregulation of hsa_circ_0058124 is correlated with unfavorable prognosis in PTC}

Quantitative real-time PCR analysis was conducted to detect hsa_circ_0058124 expression patterns in 92 paired PTC tissue samples. The hsa_circ_0058124 expression was significantly upregulated in most tumor tissues (63/ 92, 68.48\%) when compared with the adjacent normal 


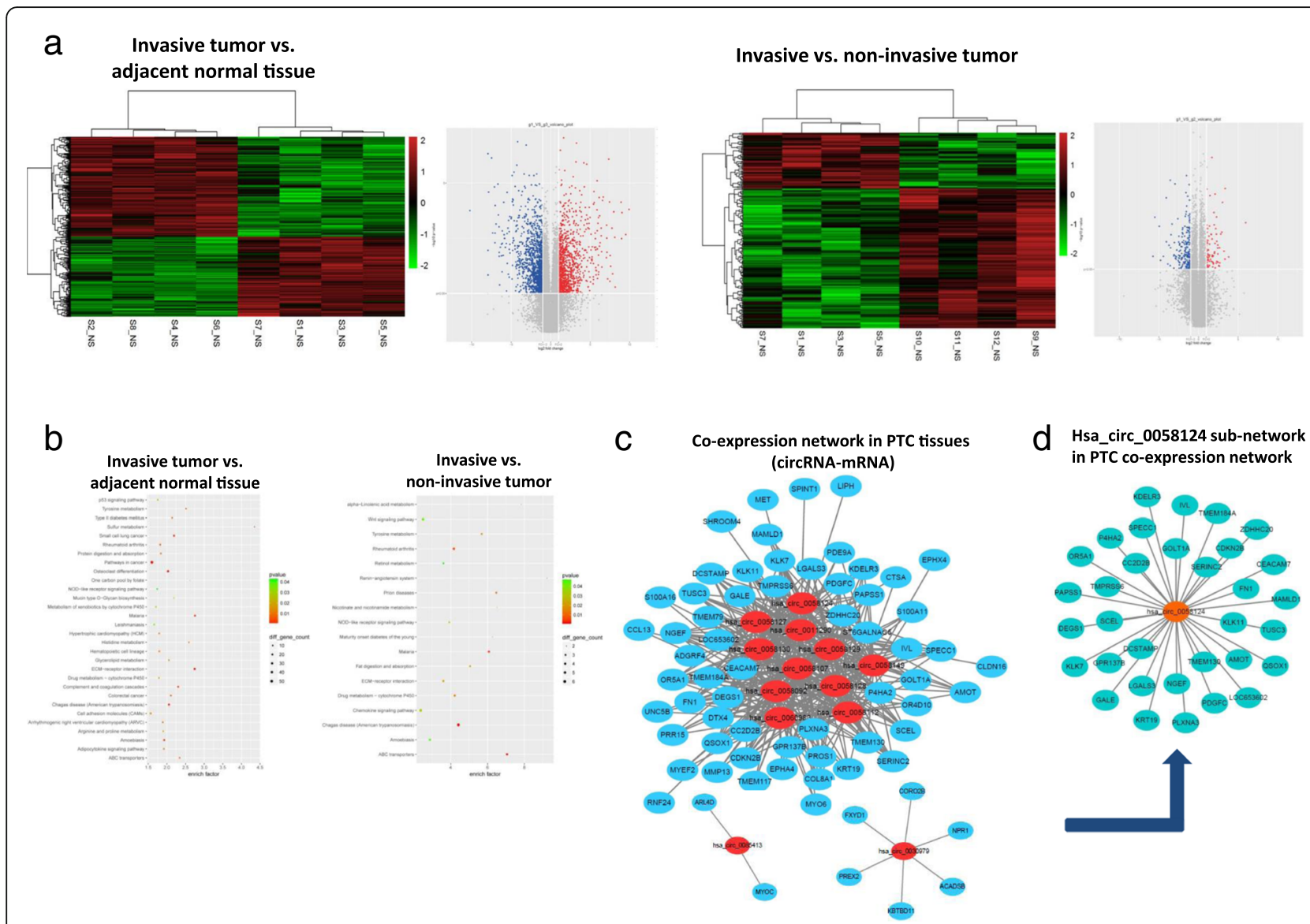

Fig. 2 The mRNA profiling and coexpression network of circRNA-mRNA interactions in PTC. a, b Hierarchical clustering analysis and KEGG analysis of the mRNA expression profile in tumor vs. non-tumor set and in invasive vs. non-invasive tumor set. c The coexpression network map included the 13 identified significantly changed circRNAs (represented as red nodes). The blue nodes around the red nodes are the predicted mRNAs that interacted with the indicated circRNAs in the eight PTC tumor samples. d The coexpression network for hsa_circ_0058124

counterparts (Fig. 4a, b). We also investigated the clinical significance of hsa_circ_0058124 by analyzing the correlations between its expression level and clinicopathological characteristics. The median value of the relative expression of hsa_circ_0058124 in PTC tissues was 1.514 , and this was used as a cut-off value to divide the patients into two groups: a high hsa_circ_0058124 relative expression $(\geq 1.514 ; n=46)$ and a low hsa_circ 0058124 relative expression $(<1.514 ; n=46)$. The clinicopathological characteristics of the 92 PTC patients are shown in Table 1 and Fig. 4. The analysis results showed that hsa_circ_0058124 expression level was significantly higher in patients with large tumor size, advanced stage, extrathyroidal extension, lymph node metastasis, or distant metastasis (Table 1, Fig. 4c). No significant correlation was noted between hsa_circ_0058124 expression and age, gender, or multifocality $(p>0.05$; Table 1$)$. These results suggest that hsa_circ_0058124 was upregulated in PTC and correlated with unfavorable prognosis. Hsa_circ_0058124 might therefore be considered as a novel prognostic biomarker.
Hsa_circ_0058124 loss of function dramatically impairs the PTC cell malignant phenotypes in vitro

We further studied the functional role of hsa_circ_0058124 in PTC cell lines by designing two siRNA oligonucleotides that would target the unique backsplice junction. These backsplice junction-specific siRNAs successfully decreased hsa_circ_0058124 expression but did not affect the linear FN1 mRNA level in TPC-1 and K1 cells (Fig. 5a). We then observed that hsa_circ_0058124 knockdown significantly decreased the cell viability determined by CCK- 8 assays (Fig. 5b). Similar results were obtained with the EdU assays, as the percentage of EdU positive cells was significantly decreased when compared with the control group (Fig. 5c). Similarly, colony formation assays revealed that hsa_circ 0058124 knockdown greatly attenuated the numbers of visible colonies (Fig. 5d). Transwell migration and invasion assays demonstrated that hsa_circ_0058124 knockdown impaired the migration and invasion ability (Fig. 6a), and the wound-healing assays showed that hsa_circ 0058124 silencing significantly suppressed the migration (Fig. 6b). Further flow cytometry investigations of 


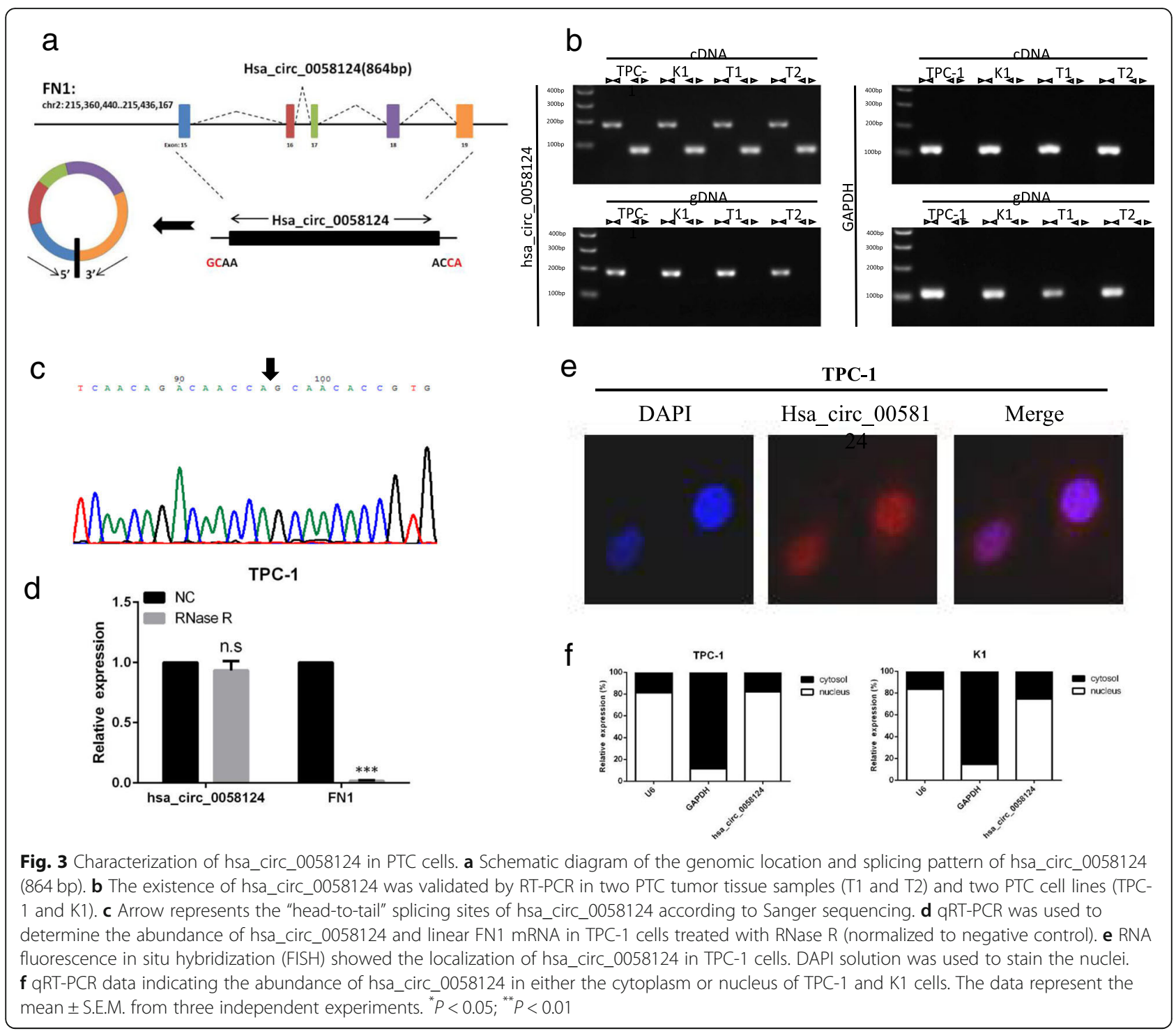

the effects of hsa_circ_0058124 on cell cycle and apoptosis revealed the percentages of apoptotic cells significantly increased after hsa_circ_0058124 knockdown in TPC-1 cells (Fig. 6c), while the proportions of cells in each cell cycle phase were not significantly different between the si-hsa circ_0058124 group and the control group (Fig. 6d).

\section{The NOTCH3 signaling pathway is a functional} downstream pathway of hsa_circ_0058124

We investigated the potential functional signaling pathways related to hsa_circ_0058124 in PTC using unbiased transcriptome profiling by RNA sequencing in TPC- 1 cells transfected with si-hsa_circ_0058124. The depletion of hsa_circ_0058124 affected the expression levels of 457 genes: 239 genes were upregulated and 218 genes were downregulated (q-value $<0.05$, |fold change $\mid>2$, Fig. 7a). The KEGG pathway enrichment analysis showed that hsa_circ_0058124 affected several downstream signaling pathways involved in cell proliferation and metastasis, including the Notch, TNF, and FoxO signaling pathways (Fig. 7b). Since the Notch signaling pathway ranked first above all signaling pathways and plays an essential role in cell migration and invasion [30], we investigated this signaling pathway as a potential functional downstream pathway for hsa_circ_0058124. GSEA analysis showed that the gene set of KEGG_NOTCH_SIGNALING_PATHWAY was significantly enriched in si-hsa_circ_0058124 group compared to si-NC group, indicating that this gene set could be significantly associated with hsa_circ_0058124 expression in PTC (Fig. 7c-d). We further validated the related genes in the NOTCH signaling pathways and found that the NOTCH3/HES1 axis showed the most significant differential expression in the pathway [NOTCH4 and MAML3 were excluded because of too low 

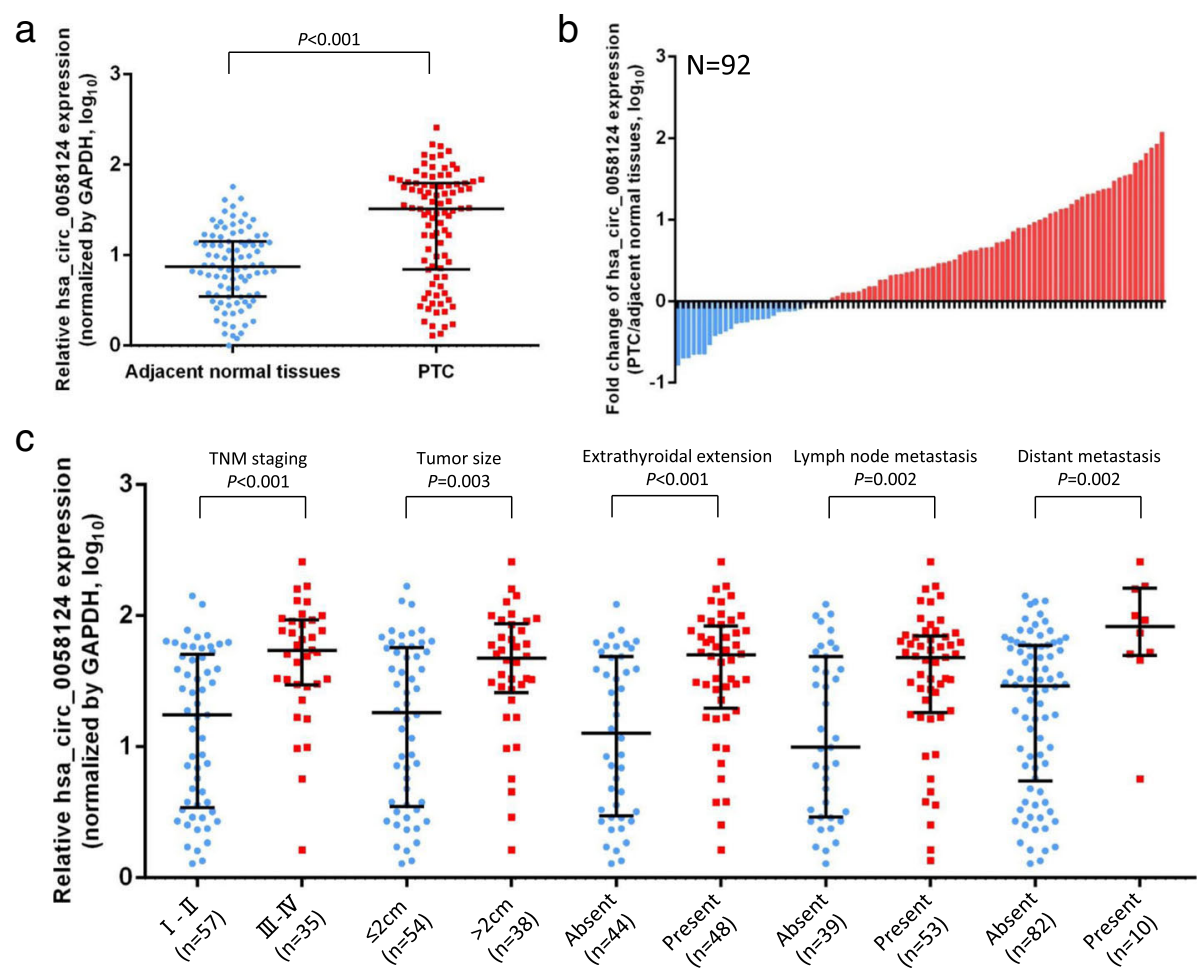

Fig. 4 Hsa_circ_0058124 is associated with clinicopathological characteristics in patients with PTC. a The copy numbers of hsa_circ_0058124 were determined by qRT-PCR in 92 pairs of PTC tissues and adjacent normal tissues. b Fold change of hsa_circ_0058124 copy number variations in 92 paired tissues. c Clinical significance of hsa_circ_0058124 in patients with PTC; high hsa_circ_0058124 expression was positively correlated with advanced stage, tumor size, extrathyroidal extension, lymph node metastasis, and distant metastasis. Values are expressed as the median with interquartile range in $(\mathbf{a}, \mathbf{c})$

expression (CT value>30)] (Fig. 7e). We also verified the NOTCH3 and HES1 protein levels by western blotting and confirmed that NOTCH3 pathway dramatically negatively related to hsa_circ_0058124 (Fig. 7f). Taken together, these findings demonstrated that hsa_circ_ 0058124 is an oncogenic driver that function by downregulating the NOTCH3 signaling pathway in PTC.

\section{Hsa_circ_0058124 affects the NOTCH3 pathway via miR- 218-5p/NUMB}

Given that circRNAs have been reported to function as miRNA sponges, we speculated that hsa_circ_0058124 might regulate the NOTCH3 pathway by sponging miRNAs. Western blottings have indicated that the NOTCH3 pathway is significantly downregulated by hsa_circ 0058124, and NUMB was reported to be a strong suppressor of the NOTCH pathway (Fig. 8a) [32, 33], we hypothesized that hsa_circ_0058124 may upregulate NUMB by sponging miRNA, which resulted in the NOTCH3 pathway suppression. Two powerful web tools, CircInteractome [34] and starBase [35], were employed to predict the potential miRNA binding partners of hsa_circ_0058124 and NUMB. As shown in Fig. 8b, we further performed Venn diagram to intersect the predicted target miRNAs and narrowed the number of miRNAs down to 7 (miR665, miR-513a-5p, miR-133a-3p, miR-3064-5p, miR-2185p, miR-4784, miR-133b). Dual luciferase assays were conducted to validate whether these miRNAs could directly target hsa_circ_0058124 and NUMB. Luciferase reporters were cotransfected with each miRNA mimic into $293 \mathrm{~T}$ cells. We found that 2 miRNAs (miR-665, miR-218-5p) significantly inhibited the luciferase reporter activity (Fig. 8c), suggesting that hsa_circ_0058124 could bind to these miRNAs. The pmiR-RB-Report vector containing 3'-UTR regions of NUMB cotransfected with miR-218-5p mimic resulted in a remarkable decrease in the Renilla/firefly ratio compared with the control vector, but not in the NUMB 3'-UTR vector cotrasfected with miR-665 (Fig. 8d), suggesting that miR-218-5p could directly bind to NUMB. Concordantly, the protein level of NUMB was significantly reduced in cells transfected with miR-218-5p mimic when compared with control cells (Fig. 8e). The results above indicated that miR-218-5p could bind both hsa_circ_0058124 and NUMB directly, and modulated NUMB expression. To further verify the miR-218-5p binding sites in hsa_circ_0058124 and NUMB, mutant (mut) and wild-type (wt) luciferase reporter constructs were transfected into TPC-1 cells together with 
Table 1 Correlation between hsa_circ_0058124 expression and clinicopathological features of 92 PTC patients

\begin{tabular}{|c|c|c|c|c|}
\hline \multirow[t]{2}{*}{ Characteristics } & \multirow[t]{2}{*}{$N$} & \multicolumn{2}{|c|}{$\begin{array}{l}\text { Relative hsa_circ_0058124 } \\
\text { expression }\end{array}$} & \multirow[t]{2}{*}{$P$ value $^{t}$} \\
\hline & & High & Low & \\
\hline Age (years) & & & & 0.210 \\
\hline$<55$ & 44 & 19 & 25 & \\
\hline$\geq 55$ & 48 & 27 & 21 & \\
\hline Gender & & & & 0.402 \\
\hline male & 42 & 23 & 19 & \\
\hline female & 50 & 23 & 27 & \\
\hline TNM staging & & & & $0.005^{*}$ \\
\hline$|-| \mid$ & 57 & 22 & 35 & \\
\hline III-IV & 35 & 24 & 11 & \\
\hline Tumor size & & & & $0.034^{*}$ \\
\hline$\leq 2 \mathrm{~cm}$ & 54 & 22 & 32 & \\
\hline$>2 \mathrm{~cm}$ & 38 & 24 & 14 & \\
\hline \multicolumn{5}{|l|}{ Extrathyroidal extension } \\
\hline Present & 48 & 29 & 19 & $0.037^{*}$ \\
\hline Absent & 44 & 17 & 27 & \\
\hline \multicolumn{5}{|l|}{ Multifocality } \\
\hline Present & 25 & 14 & 11 & 0.482 \\
\hline Absent & 67 & 32 & 35 & \\
\hline Lymph node metastasis & & & & $0.020^{*}$ \\
\hline Present & 53 & 32 & 21 & \\
\hline Absent & 39 & 14 & 25 & \\
\hline Distant metastasis & & & & $0.007^{*}$ \\
\hline Present & 10 & 9 & 1 & \\
\hline Absent & 82 & 37 & 45 & \\
\hline
\end{tabular}

${ }^{\dagger}$ Pearson's chi-squared test

${ }^{*} P<0.05$

miR-218-5p mimics or controls (miR-NC). miR-218-5p mimics significantly inhibited luciferase activity in cells transfected with wild-type constructs, but luciferase activity was not affected in cells transfected with mutant constructs (Fig. 8f, g), indicating that miR-218-5p directly targets hsa_circ_0058124 and NUMB. Then, we determined the expression of miR-218-5p and NUMB by qRTPCR in 20 pairs of PTC and matched normal tissues. We observed that miR-218-5p expression was downregulated in PTC tissues compared with normal tissues, while the expression of NUMB was significantly upregulated in PTC tissues (Additional file 2: Figure S3). There was also a negative correlation of hsa_circ_0058124 and miR-218-5p, similar to that of miR-218-5p and NUMB (Additional file 2: Figure S4). Moreover, miR-218-5p expression was significantly upregulated with hsa_circ_0058124 knockdown (Fig. 8h), and the expression of NUMB at both the mRNA (Fig. 8i) and protein level (Fig. 8j) were significantly decreased after hsa_circ_0058124 silencing in TPC-1 and $\mathrm{K} 1$ cells. As shown in Fig. 8k, we demonstrated that the NOTCH3 pathway was regulated by hsa_circ_0058124/ miR-218-5p/NUMB axis. NOTCH3 pathway was significantly activated by hsa_circ_0058124 suppression, while sihsa_circ_0058124 induced NOTCH3 pathway activation was attenuated with miR-218-5p inhibition or NUMB overexpression. These results indicated that hsa_circ 0058124 suppress NOTCH3 pathway via miR-218-5p/ NUMB. Furthermore, miR-218-5p inhibition or NUMB overexpression significantly attenuated the si-hsa_circ 0058124-induced inhibition of growth (Fig. 81), migration and invasion (Fig. $8 \mathrm{~m}$ ). Taken together, these results suggest that hsa_circ_0058124 promotes PTC progression through miR-218-5p/NUMB axis to suppress NOTCH3 pathway.

\section{Hsa_circ_0058124 promotes PTC progression through the NOTCH3/GATAD2A axis}

The GATA family is known to interact with the NOTCH signaling pathway [36-38]. Our mRNA-sequence data also showed GATAD2A was significantly dysregulated in sihsa_circ_0058124 group. Therefore, we questioned whether hsa_circ_0058124 may promote PTC progression through down-regulating NOTCH3/GATAD2A axis. Consistently, hsa_circ_0058124 silencing significantly increased the expression of Notch3 and GATAD2A at the protein level. In addition, blocking the NOTCH3 pathway with si-NOTCH3 also downregulated GATAD2A, even with suppressed hsa circ_0058124, while suppression of GATAD2A did not significantly affect the NOTCH3 expression in TPC-1 cells (Fig. 9a). These findings indicated that the suppression of GATAD2A by hsa_circ_0058124 depends on NOTCH3.

We confirmed whether the oncogenic effects of hsa circ_0058124 knockdown were mediated by NOTCH3/ GATAD2A axis in PTC cells by cotransfecting si-hsa_circ 0058124 and si-NOTCH3 or si-GATAD2A into two PTC cell lines. Subsequent functional experiments showed that the suppression of NOTCH3 or GATAD2A could partly impair the si-hsa_circ_0058124-induced inhibition of growth (Fig. 9b-c), migration, and invasion (Fig. 9d). Thus, suppression of NOTCH3 or GATAD2A could restore the malignant behavior of hsa_circ_0058124 knockdown. However, the results also indicated that suppression of NOTCH3 or GATAD2A only partly impaired the si-hsa circ_0058124-induced inhibition of the malignant behavior in PTC cells, which suggested that NOTCH3/GATAD2A axis might just be one of the major downstream target genes regulated by hsa_circ_0058124.

To investigate the GATAD2A expression levels in PTC tissues, we collected 5 PTC tissues with paired adjacent normal tissues, and measured the expression levels of GATAD2A by IHC assays. The results showed that GATAD2A expression was much lower in the PTC tissues 


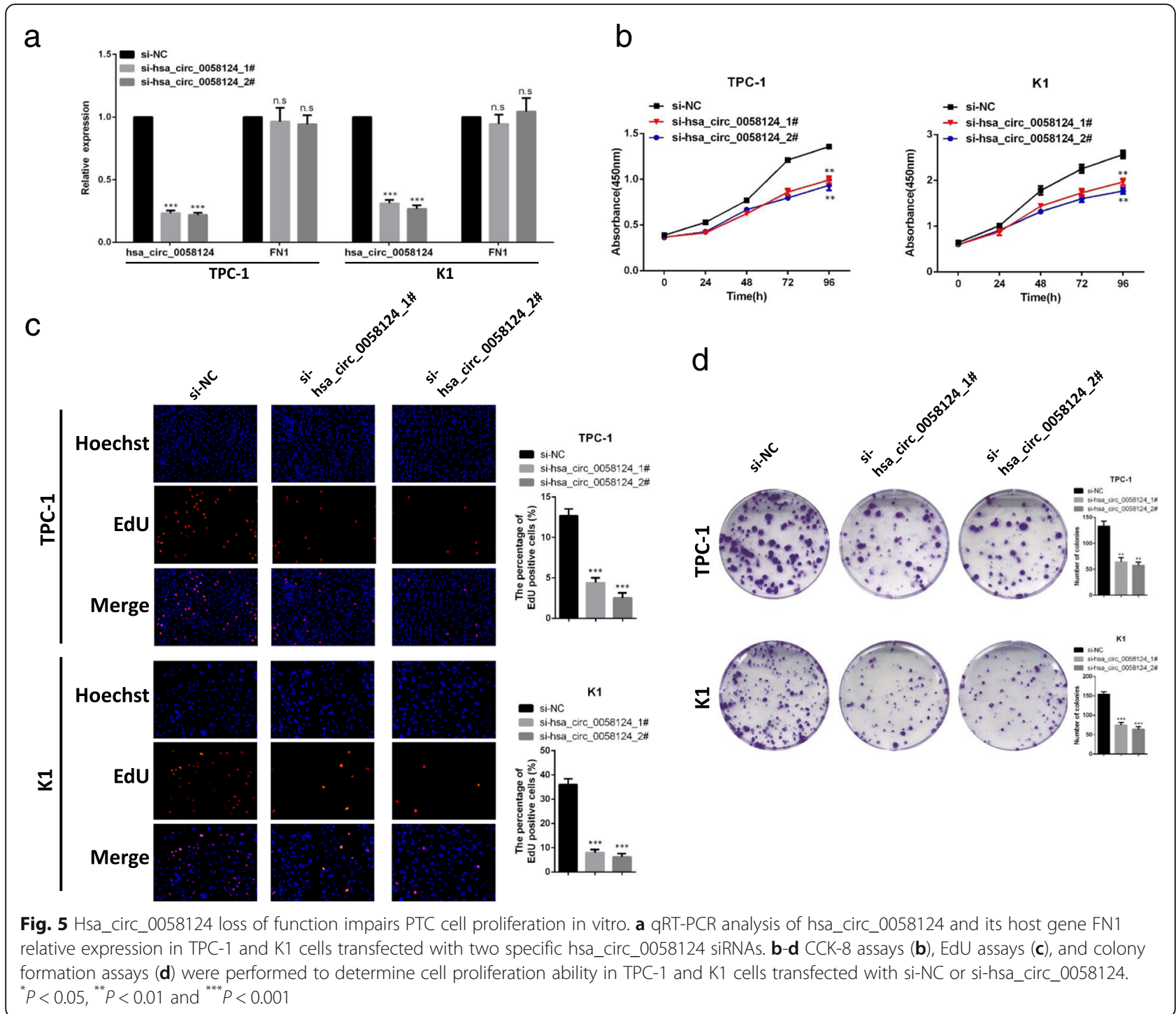

compared with adjacent normal tissues (Fig. 9e). We also validated the differential expression of GATAD2A in PTC by examining IHC staining data of thyroid tumor tissues and normal thyroid tissues from the Human Protein Atlas. The fraction of samples with high, medium, low, or not detected levels of protein expression were provided by the blue-scale color-coding. As shown in Fig. 9f, all 4 thyroid tumor samples showed medium expression of GATAD2A, while all 3 normal samples showed high expression. Collectively, these data indicated that the underlying mechanism by which hsa_circ_0058124 promotes PTC progression is associated with a suppression of the NOTCH3/GATAD2A axis.

The effects of hsa_circ_0058124/NOTCH3/GATAD2A axis in PTC tumor formation in vivo

To further explore the effects of hsa_circ_0058124/ NOTCH3/GATAD2A axis on PTC cells in vivo, we injected nude mice (aged 5-6 weeks, 5 in each group) with TPC-1 cells transfected with indicated shRNAs $\left(3 \times 10^{6}\right.$ cells $)$ subcutaneously for the control group, the hsa_circ_0058124 stably knocked down group, the NOTCH3 stably knocked down group, the GATAD2A stably silencing group, the stable knock down hsa circ_0058124 with NOTCH3 inhibition group or the stable knock down hsa_circ_0058124 with suppressing GATAD2A group. Increases in tumor sizes were examined and recorded in each group every 3 days. At 27 days after TPC-1 cell injection, the mice were humanely euthanized, and the tumor volume and weight of the dissected tumors were measured. As demonstrated in Fig. 10a-d, the in vivo tumor formation assay suggested that hsa_circ_0058124 knockdown dramatically inhibited tumor growth when compared to the negative control group, whereas suppression of NOTCH3 or GATAD2A partly abolished this reduction of tumor growth by hsa 


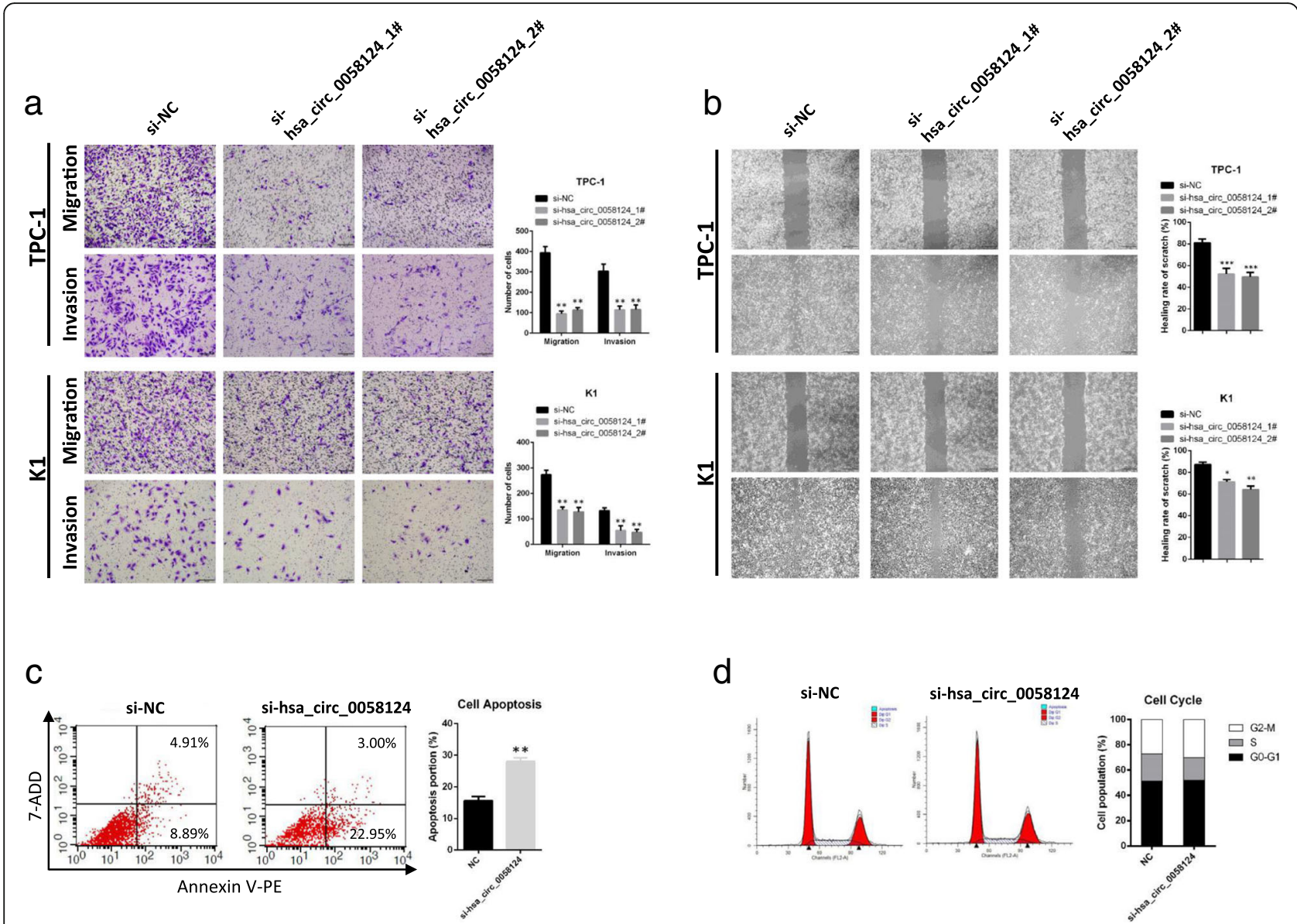

Fig. 6 Hsa_circ_0058124 affects the migration, invasion abilities, and apoptosis of PTC cells in vitro. a Representative images and quantification results of cell migration and invasion abilities of TPC-1 and K1 cells harboring control or hsa_circ_0058124 siRNAs. $\mathbf{b}$ Scratch wound assays in hsa_circ_0058124-deficient PTC cells and corresponding controls. c, d Flow cytometry assays showed the rate of apoptosis (c) and the cell cycle distributions (d) in TPC-1 cells transfected with si-NC or si-hsa_circ_0058124. ${ }^{*} P<0.05,{ }^{* *} P<0.01$ and ${ }^{* * *} P<0.001$

circ_0058124 knockdown. Taken together, these findings demonstrated that hsa_circ_0058124 is an oncogenic driver that functions by suppressing the NOTCH3/ GATAD2A axis in PTC in vivo.

\section{Discussion}

The incidence of PTC has been rapidly increasing over the past few decades. Although the prognosis of patients with PTC is generally good, tumor invasiveness and metastasis are the major risk factors that lead to poor prognosis $[5,6,39]$. Cancer cell migration and invasion are heterogeneous and adaptive processes that include changes in cell morphology, loss of cell polarity, and gains in cell motility that ultimately result in translocation. Cancer invasion is what lies behind the metastatic that remains the major cause of mortality in patients with cancer $[9,30]$. Therefore, the major challenges in PTC research are elucidating the mechanisms underlying the invasiveness and metastasis of PTC and developing novel clinical strategies that could intervene the progression of PTC.
In the present study, we investigated the circRNAs that may contribute to the tumorigenesis and invasiveness in PTC by selecting eight PTC samples (including four invasive extrathyroidal extension tumor samples with metastasis and four non-invasive tumor samples with no metastasis) and four matching adjacent normal tissue samples. We then divided these tissue samples into two sets (invasive tumor vs adjacent normal tissue set and invasive vs non-invasive tumor set) and used ceRNA microarray analysis to identify dysregulated circRNAs. These upregulated and downregulated circRNAs and mRNAs were located on different chromosomes and formed a coexpression network for PTC. Combined with the results of previous studies showing that circRNAs are strongly related to thyroid cancer [20, 21], we found that specific circRNAs are candidate biomarkers and molecular targets for PTC.

Our investigations identified a novel circRNA hsa_circ 0058124 in PTC pathogenesis. We demonstrated that hsa circ_0058124 was present in PTC tissues and cell lines and was resistant to RNase R. We found that hsa_circ_0058124 


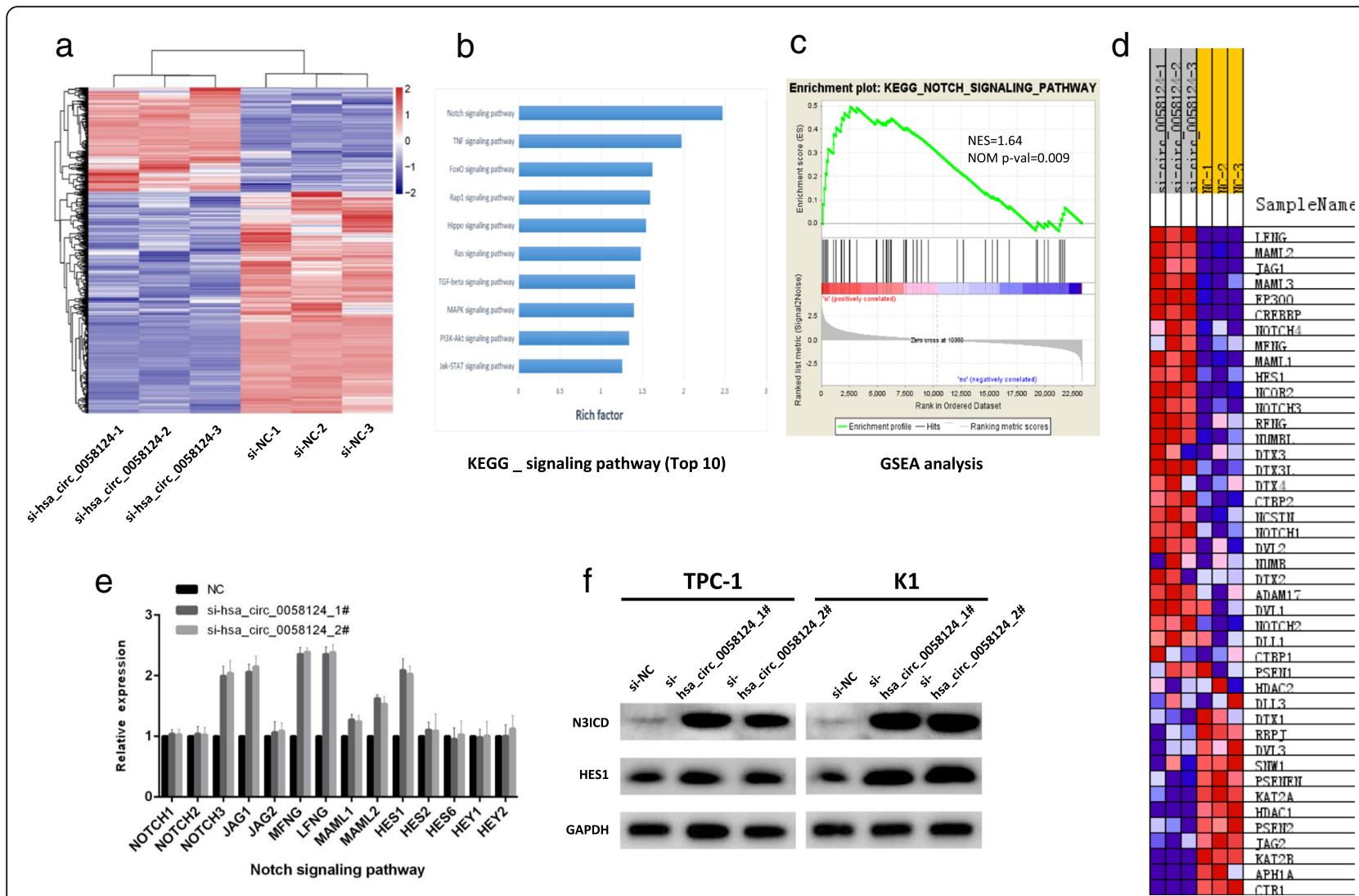

Fig. 7 Hsa_circ_0058124 promotes PTC progression through NOTCH3 signaling pathway. a Heatmap representation of the significantly differentially expressed genes from RNA-seq data after treatment with the indicated siRNAs. $\mathbf{b}$ KEGG enrichment focused on a set of signaling pathways after hsa_circ_0058124 silencing, summarized based on the enrichment score. c, d GSEA analysis of the "KEGG_NOTCH_SIGNALING_PATHWAY" gene set in the si-NC versus si-hsa_circ_0058124 group in TPC-1 cells. The top portion of the figure plots the enrichment scores for each gene, whereas the bottom portion of the plot shows the value of the ranking metric moving down the list of ranked genes. Y-axis: value of the ranking metric; X-axis: the rank for all genes. NES, normalized enrichment score. $\mathbf{e}$ The mRNA levels of the related genes in the NOTCH signaling pathways in TPC-1 cells treated with si-hsa_circ_0058124.f NOTCH3 and HES1 protein levels in TPC-1 and K1 cells with hsa_circ_0058124 deficiency. Values are expressed as the mean \pm S.E.M, $n=3$ in a, b, c, d. ${ }^{*} P<0.05,{ }^{* *} P<0.01$ and ${ }^{* * *} P<0.001$

was frequently upregulated in PTC tissues and its upregulation was also associated with poor prognosis. The results also revealed hsa_circ_0058124 significantly promoted the growth, proliferation, invasiveness, and migration of PTC in vivo and in vitro. These findings are of great significance because this is the first study to demonstrate the differentially expression circRNAs of invasive PTC compared with ordinary PTC. The results also pointed to critical roles of a previously uncharacterized circRNA, hsa_circ_0058124, involved in the migratory and invasive capacities of PTC, and suggested that hsa_circ_0058124 might function as a tumor oncogene and an unfavorable prognostic biomarker. Our findings also indicated an involvement of the $\mathrm{NOTCH}$ pathway.

The NOTCH pathway is highly conserved in evolution and plays important roles in the stem cell maintenance, cellular differentiation, and determination [40]. Canonical $\mathrm{NOTCH}$ signaling consists of four $\mathrm{NOTCH}$ receptors (NOTCH1-4) and their ligands (Delta-like 1, 3, and 4 and
Jagged 1 and 2). In mammals, all NOTCH receptors have a similar structure consisting of extracellular, transmembrane, and intracellular subunits [41]. In the most widely accepted core pathway of $\mathrm{NOTCH}$ activation, ligand binding unfolds the negative regulatory region to permit the first cleavage through metalloproteinases of the ADAM family. Subsequently, the $\gamma$-secretase complex, as the second cleavage mediator, performs an intramembrane cleavage releasing the NOTCH intracellular domain (NICD), which then translocates to the nucleus [42]. Following NOTCH activation, downstream targets, such as the hairy and enhancer of split (HES) family and the hairy-related transcription factor, are expressed and in turn orchestrate the NOTCH-induced nuclear reprogramming [43]. NOTCH signaling pathway participated in the development of a variety of cancers by regulating the tumor microenvironment, tumorigenesis, progression, angiogenesis, invasion and metastasis [44, 45]. $\mathrm{NOTCH}$ has also been implicated in cancer cell metabolism, survival and drug resistance; maintenance of cancer stem 


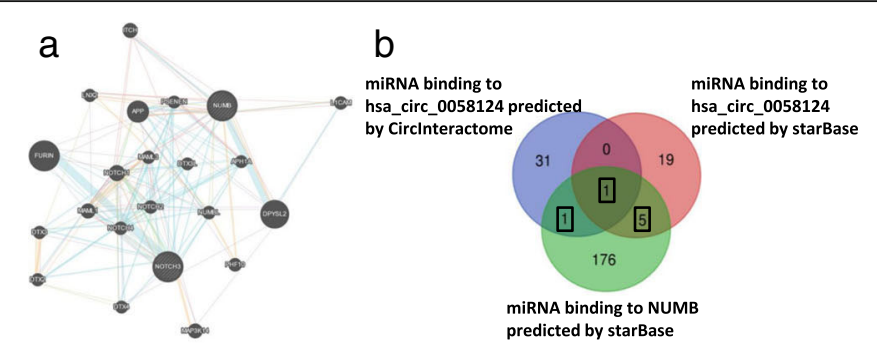

\section{C}
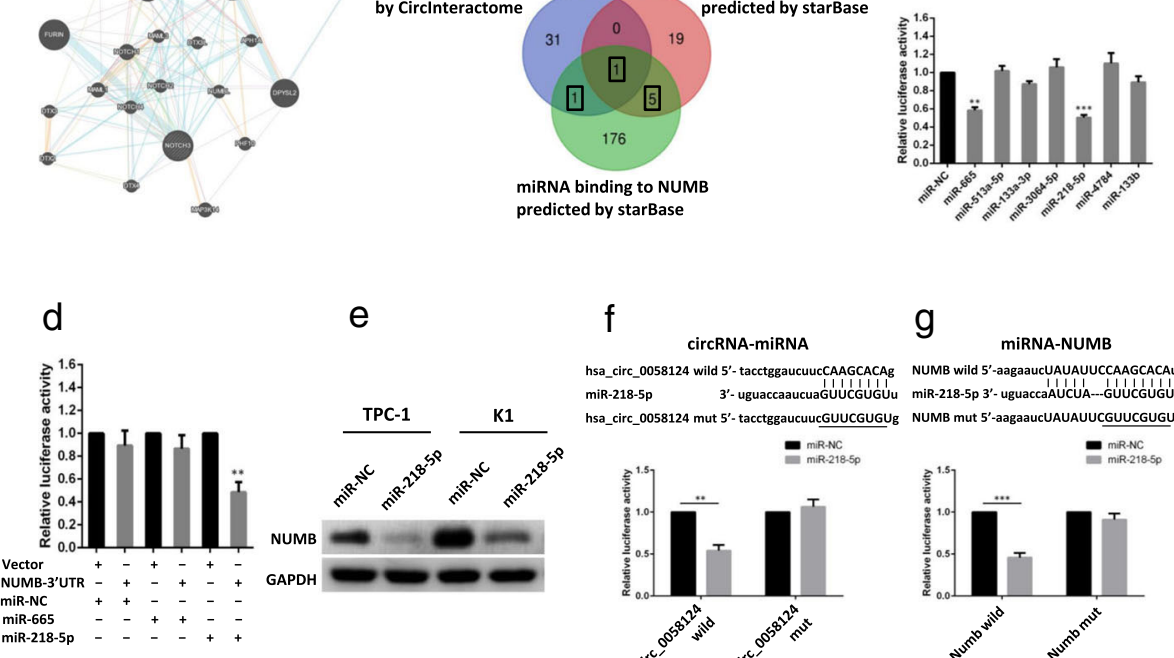

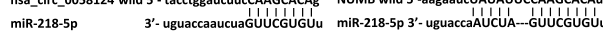
hsa_circ_0058124 mut 5' - tactggaucuucGUUCGUGUg NUMB mut 5'-aagaaucUAUAUUCGUUCGUGUU
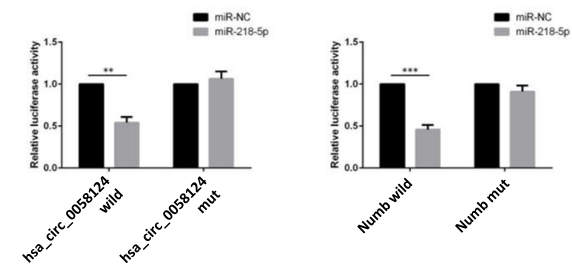

$\mathrm{h}$

i
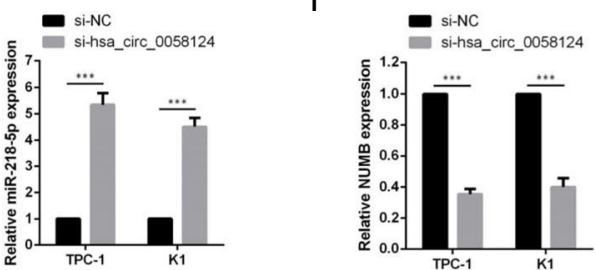

j
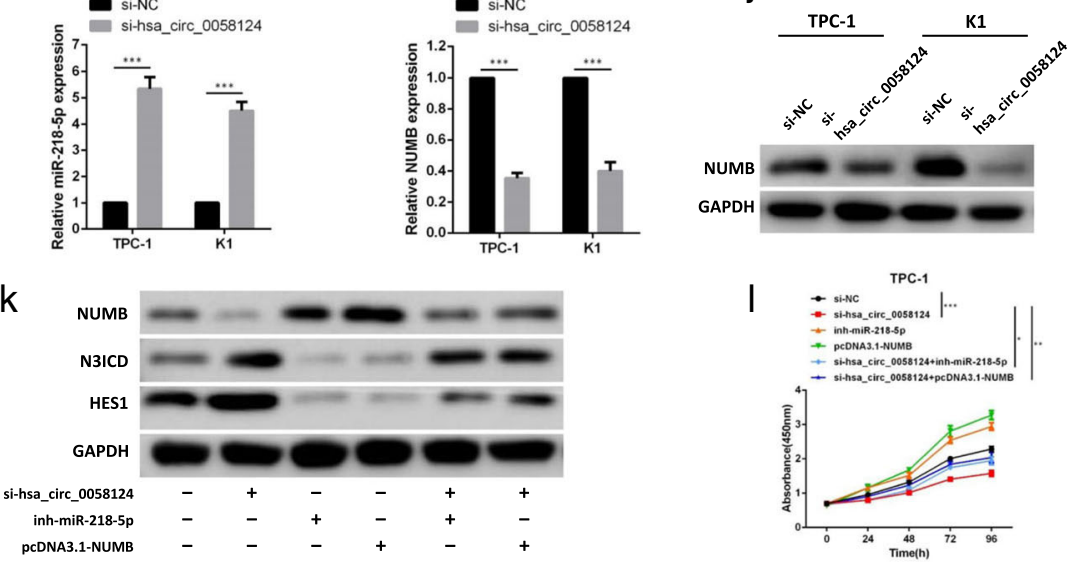

TPC.1

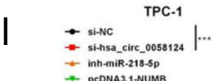

$=$ inh-miR.212:-5p
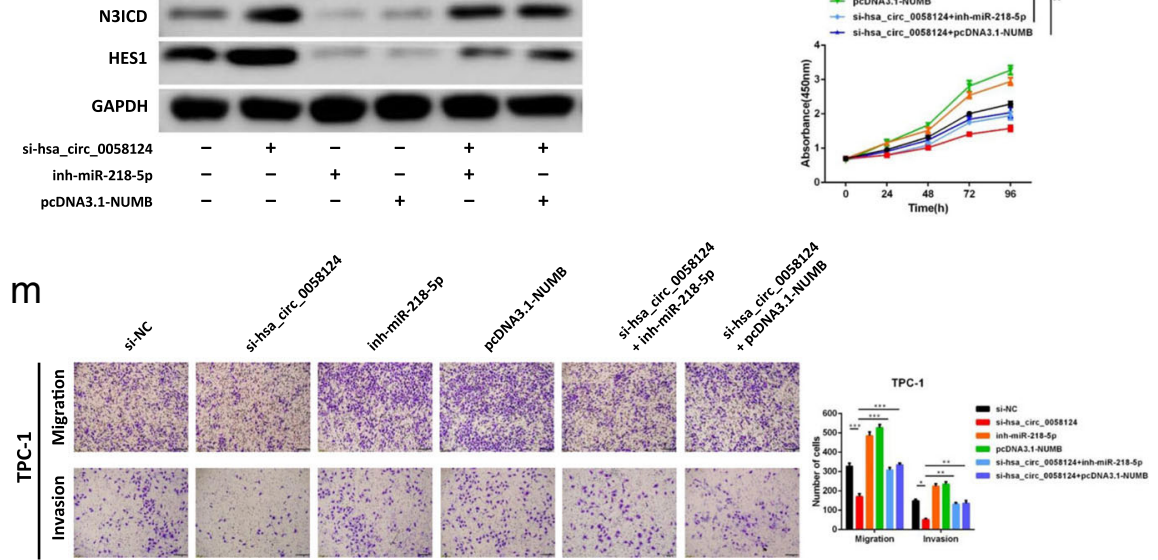

Fig. 8 Hsa_circ_0058124 affects the NOTCH3 pathway via miR-218-5p/NUMB. a The interaction network between the NOTCH3 pathway and hsa_circ_0058124 using GeneMANIA web tool [31]. b Venn diagram showing the mutual putative target genes of hsa_circ_0058124 and NUMB predicted by Circlnteractome and starBase. c Dual-luciferase assays showing the luciferase activity of the pmiR-RB-Report-hsa_circ_0058124 vector in 293 T cells cotransfected with indicated miRNA mimics. $\mathbf{d}$ Dual-luciferase assays confirmed the interaction of NUMB with miR-218-5p. e NUMB protein level after transfection with miR-NC or miR-218-5p. $\mathbf{f}, \mathbf{g}$ mutant (mut) and wild-type (wt) luciferase reporter constructs of hsa_circ_0058124 (f) or NUMB (g) were transfected into TPC-1 cells together with miR-218-5p mimics or controls (miR-NC). Dual-luciferase assays were conducted to detect relative luciferase activity. $\mathbf{h}$ Expression level of miR-218-5p in TPC-1 and K1 cells infected with si-hsa_circ_0058124 or negative control (si-NC) were measured by qRT-PCR. i-j NUMB mRNA (i) and protein level (j) in TPC-1 and K1 cells transfected with si-hsa_circ_0058124 or si-NC. $\mathbf{k}$ Western blot assays were performed to measure the NOTCH3 and HES1 protein level in TPC-1 cells with indicated treatments. I-m CCK-8 assays (I) and transwell assays (m) in TPC-1 cells with indicated treatments 

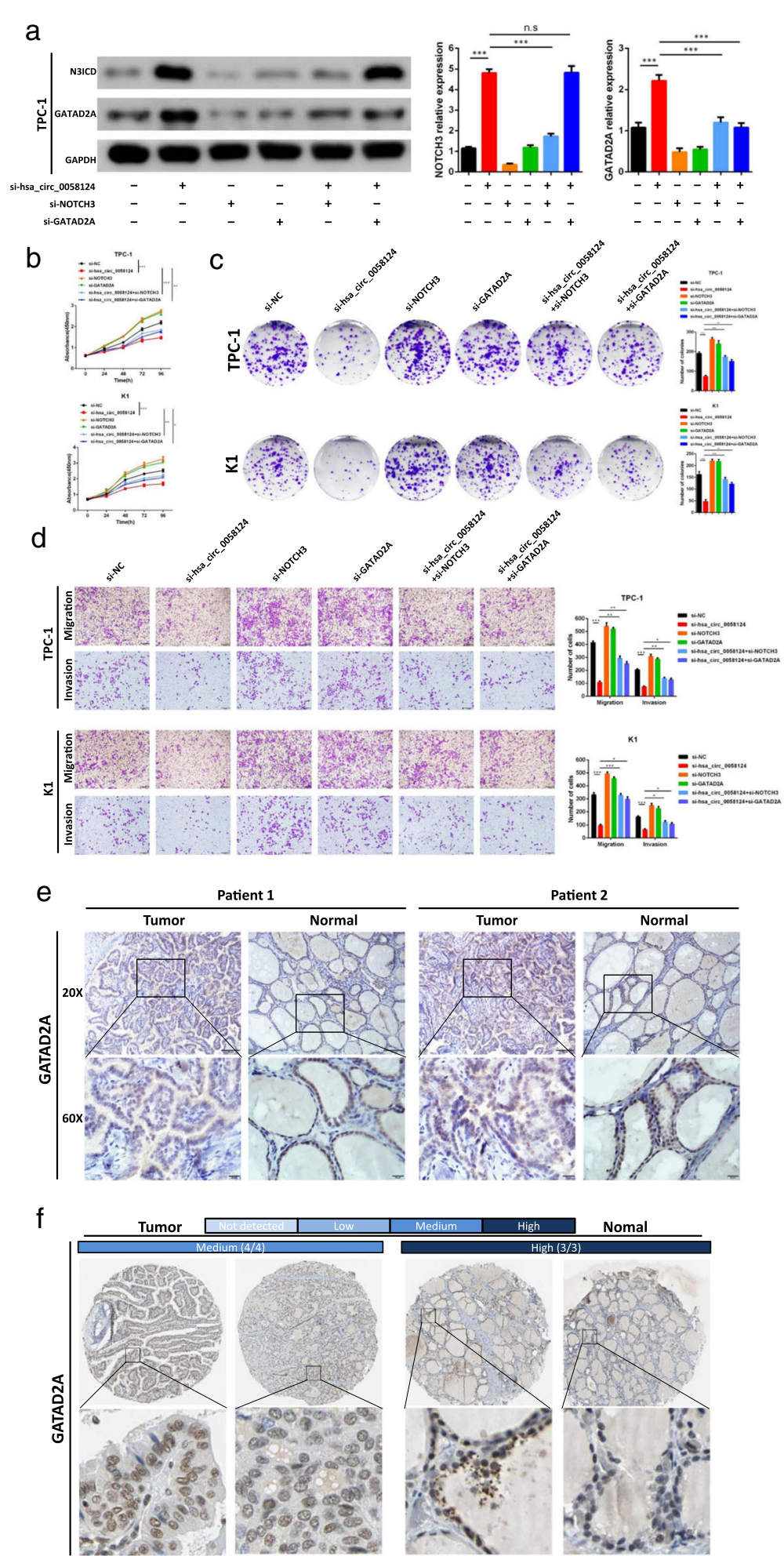

Fig. 9 (See legend on next page.) 
(See figure on previous page.)

Fig. 9 The oncogenic hsa_circ_0058124/NOTCH3/GATAD2A axis in PTC cells. a Expression of Notch3 and GATAD2A in TPC-1 cells at protein level analyzed by western blot, after transfection with the indicated siRNAs. b, c The CCK-8 assays (b) and colony formation assays (c) were used to evaluate the cell growth after transfection with si-hsa_circ_0058124 or co-transfected with si-hsa_circ_0058124 and si-GATAD2A or si-NC in PTC cells. d Transwell assays were applied to evaluate the migration and invasion of PTC cells after transfection with si-hsa_circ_0058124 or co-transfected with sihsa_circ_0058124 and si-GATAD2A or si-NC. e Immunohistochemistry analysis of GATAD2A protein levels in PTC tissues. Representative images were shown. Scale bar, $100 \mu \mathrm{m}$. f Representative images for the expression of GATAD2A in thyroid tumor tissues and normal thyroid tissues are shown with the fraction of samples with antibody staining/protein expressions evaluated as high, medium, low, or not detected based on the blue-scale color coding. Data are presented as the mean \pm S.E.M., analyzed using independent samples student's t-test. ${ }^{*} P<0.05,{ }^{* *} P<0.01$ and ${ }^{* * *} P<0.001$

cells; epithelial-mesenchymal transition; and genomic instability [46]. Our unbiased transcriptome profile in TPC1 cells transfected with si-hsa_circ_0058124 by RNA sequencing indicated that the $\mathrm{NOTCH} 3$ signaling pathway is a functional downstream target for hsa_circ_0058124. As for how hsa_circ_0058124 modulate NOTCH3 pathway, we assumed that hsa_circ_0058124 suppress NOTCH3 pathway by sponging miRNA and then upregulating NUMB expression, on basis of circRNA often acting as miRNA sponge and NUMB as a strong suppressor of the NOTCH pathway. Luciferase assays have been shown efficiently identify the precise and authentic interactions between miR-218-5p and hsa_circ_0058124 or NUMB. Our results demonstrated that hsa_circ_0058124 suppresses the NOTCH3 pathway via miR-218-5p/NUMB. The results further suggested an involvement of GATAD2A.

GATAD2A (GATA zinc finger domain containing 2A) is a subunit of the nucleosome remodeling and histone
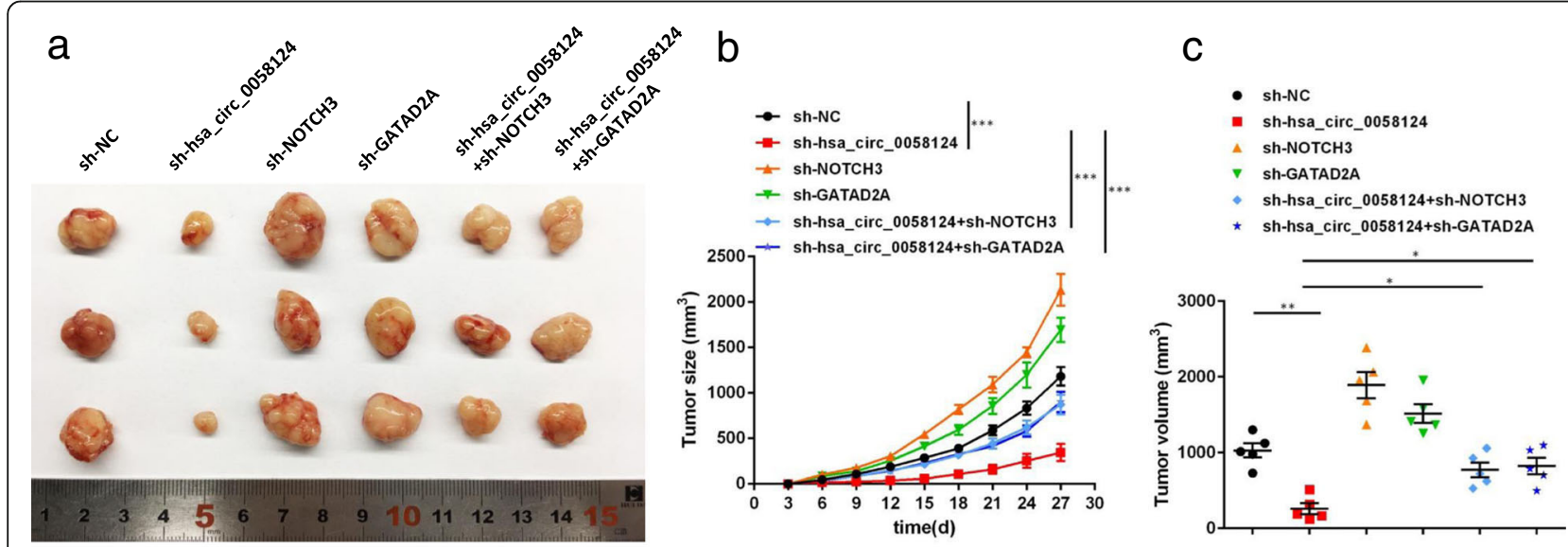

d

e
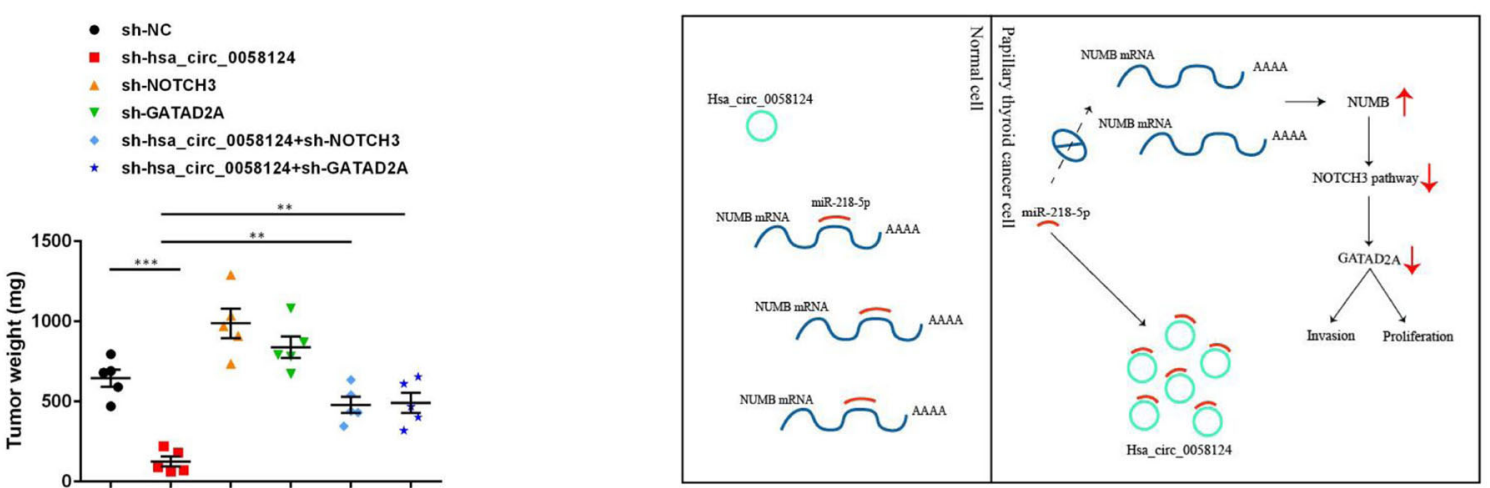

Fig. 10 Hsa_circ_0058124/NOTCH3/GATAD2A axis promotes PTC tumor formation in vivo. a TPC-1 cells with indicated transfection $\left(3 \times 10^{6}\right.$ cells per mouse, $n=5$ for each group) were inoculated into nude mice to establish subcutaneous xenograft tumors. The mice were sacrificed at 27 days. The dissected tumors were photographed. Representative macroscopic views showed the tumors in the indicated groups. $\mathbf{b}$ Tumor sizes were measured every 3 days after the tumors were palpable. $\mathbf{c}, \mathbf{d}$ Dissected tumor volumes and weights were measured at 27 days. e Schematic model of depicting hsa_circ_0058124 as an oncogene in PTC. ${ }^{*} P<0.05,{ }^{* *} P<0.01$ and ${ }^{* * *} P<0.001$ 
deacetylation (NuRD) complex, which is a key factor involved in various biological progresses including tumor growth inhibition, cellular differentiation, embryonic development and the general repression of transcription [47, 48]. A previous study reported the differential expression of GATAD2A in thyroid cancer and suggested its possible involvement in thyroid cancer progression [49]. Meanwhile, several reports have shown interactions between the GATA family and the NOTCH signaling pathway [36-38]. Therefore, we hypothesized that hsa_circ_0058124 might promote PTC progression via a NOTCH3/GATAD2A axis. Consistently, our findings demonstrated that hsa_circ 0058124 promote PTC progression by suppressing the NOTCH3/GATAD2A axis in vitro and in vivo.

In the current study, we demonstrated that hsa_circ 0058124 promotes PTC tumorigenesis and invasiveness through sponging miR-218-5p and upregulating its target gene NUMB expression, subsequently with suppression of NOTCH3/GATAD2A axis (Fig. 10e). These findings indicate a critical role for the hsa_circ_0058124/ NOTCH3/GATAD2A oncogenic axis in the tumorigenesis and invasiveness of PTC and provide a preclinical rationale for the design of novel therapeutic strategies that will target the this axis to improve the clinical outcome of patients with PTC.

\section{Conclusions}

In conclusion, our findings showed the overexpression of a novel circRNA, hsa_circ_0058124, located on 2q35 in PTC cells. Hsa_circ_0058124 acts as an oncogene and significantly promotes PTC cell proliferation, tumorigenicity, and tumor invasiveness both in vitro and in vivo. Hsa_circ_0058124 exerts its oncogenic activity by suppressing the NOTCH3/ GATAD2A axis in a miR-218-5p/NUMB dependent manner in PTC cells. This hsa_circ_0058124 is a promising prognostic indicator, and its discovery provides insight into PTC carcinogenesis and aggressiveness, and may facilitate the development of precise approaches for cancer screening and treatment.

\section{Additional files}

Additional file 1: Table S1. The primer sequences of the RNAs used in this study. Table S2. Sequence of siRNAs used in this study. Table S3. Clinical information of twelve samples from eight PTC patients included for ceRNA microarray analysis. Table S4. Differentially expressed circRNAs overlapped between the invasive vs. non-invasive tumor set and the invasive tumor vs. adjacent normal tissue set. (DOCX $21 \mathrm{~kb}$ )

Additional file 2: Figure S1. The circRNA-mRNA coexpression validated by qRT-PCR. Figure S2. Hsa_circ_0058124 expression in PTC tissues. Figure S3. The miR-218-5p and NUMB expression in PTC and matched normal tissues tissues. Figure S4. Correlation between miR218-5p and hsa_circ_0058124 or NUMB. (PPTX 1455 kb)

Additional file 3: The genomic location and splicing pattern of hsa_circ_0058124 (864 bp). (DOCX 72 kb)

\section{Abbreviations}

bp: Base pairs; ceRNA: Competing endogenous RNA; CircRNAs: Circular RNAs: EdU: 5-ethynyl-2'-deoxyuridine; ETE: Extrathyroidal extension;

FISH: Fluorescent in situ hybridization; GAPDH: Glyceraldehyde-3-phosphate dehydrogenase; GSEA: Gene Set Enrichment Analysis; KEGG: Kyoto Encyclopedia of Genes and Genomes; OD: Optical density; PTC: Papillary thyroid cancer; qRT-PCR: Quantitative real-time polymerase chain reaction; RNA-seq: RNA sequencing; shRNAs: Short hairpin RNA; siRNAs: Small interfering RNAs; TC: Thyroid cancer

\section{Acknowledgements \\ The authors thank all patients involved in this study.}

\section{Authors' contributions}

$X C$ and $Y Y$ performed experiments, analyzed data and wrote the paper; $H Y$, WC, YQ, ZY, TL, WY, WW and TY performed some experiments and analyzed data; $Y Y, Y C$ and $Y Z$ initiated the study, designed experiments and wrote the paper. All authors read and approved the final manuscript.

\section{Funding}

This study was funded by the National Natural Science Foundation (81772602, 9174210027), Jiangsu Provincial Key Research Development Program (BE2016796, BE2018750), the Six Talent Peaks Project (2016-WSW022, JY-018), the project of Nanjing Science and Technology Commission (201611006), the China Postdoctoral Science Foundation (2017M621676).

\section{Availability of data and materials}

All data and materials can be provided upon request.

\section{Ethics approval and consent to participate}

This study was approved by the Ethics Committee of Nanjing Medical University and was conducted in accordance with the government policies and the Declaration of Helsinki. Written informed consent was obtained from all patients.

\section{Consent for publication}

All authors agree for publication.

\section{Competing interests}

The authors declare that they have no competing interests.

\section{Author details}

'Department of Head and Neck Surgery, Jiangsu Cancer Hospital \& Jiangsu Institute of Cancer Research \& The Affiliated Cancer Hospital of Nanjing Medical University, Nanjing 210009, China. ${ }^{2}$ Department of Immunology, Key Laboratory of Immune Microenvironment and Disease, Nanjing Medical University, Nanjing 211166, China. ${ }^{3}$ Jiangsu Key Lab of Cancer Biomarkers, Prevention and Treatment, Collaborative Innovation Center for Cancer Personalized Medicine, Nanjing Medical University, Nanjing 211166, China. ${ }^{4}$ Jiangsu Key Laboratory of Oral Disease, Nanjing Medical University, Jiangsu 210029, China. ${ }^{5}$ Department of Oral and Maxillofacial Surgery, Affiliated Hospital of Stomatology, Nanjing Medical University, Jiangsu 210029, China. ${ }^{6}$ Department of Head and Neck Surgery, Fudan University Shanghai Cancer Center, Shanghai 200032, China. ${ }^{7}$ Department of Oncology, Shanghai Medical College, Fudan University, Shanghai 200032, China.

Received: 30 January 2019 Accepted: 10 July 2019

References

1. Kitahara CM, Sosa JA. The changing incidence of thyroid cancer. Nat Rev Endocrinol. 2016;12(11):646-53.

2. Siegel RL, Miller KD, Jemal A: Cancer statistics, 2018. 2018, 68(1):7-30.

3. Cabanillas ME, McFadden DG, Durante C. Thyroid cancer. Lancet. 2016; 388(10061):2783-95

4. Fagin JA, Wells SA Jr. Biologic and clinical perspectives on thyroid Cancer. N Engl J Med. 2016;375(11):1054-67.

5. Song E, Jeon MJ, Oh HS, Han M, Lee YM, Kim TY, Chung KW, Kim WB, Shong YK, Song DE, et al. Do aggressive variants of papillary thyroid carcinoma have worse clinical outcome than classic papillary thyroid carcinoma? Eur J Endocrinol. 2018;179(3):135-42. 
6. Jiang C, Cheng T, Zheng X, Hong S, Liu S, Liu J, Wang J, Wang S. Clinical behaviors of rare variants of papillary thyroid carcinoma are associated with survival: a population-level analysis. Cancer Manag Res. 2018;10:465-72.

7. Kunavisarut T. Diagnostic biomarkers of differentiated thyroid cancer. Endocrine. 2013;44(3):616-22.

8. Lundgren $\mathrm{Cl}$, Hall P, Dickman PW, Zedenius J. Clinically significant prognostic factors for differentiated thyroid carcinoma: a population-based, nested case-control study. Cancer. 2006;106(3):524-31.

9. Nixon IJ, Simo R, Newbold K, Rinaldo A, Suarez C, Kowalski LP, Silver C, Shah JP, Ferlito A. Management of Invasive Differentiated Thyroid Cancer. Thyroid. 2016;26(9):1156-66.

10. Teng H, Mao F, Liang J, Xue M, Wei W, Li X, Zhang K, Feng D, Liu B, Sun Z. Transcriptomic signature associated with carcinogenesis and aggressiveness of papillary thyroid carcinoma. Theranostics. 2018:8(16):4345-58.

11. Dhamija S, Menon MB. Non-coding transcript variants of protein-coding genes - what are they good for? RNA Biol. 2018;15(8):1025-31.

12. Ashwal-Fluss R, Meyer M, Pamudurti NR, Ivanov A, Bartok O, Hanan M, Evantal N, Memczak S, Rajewsky N, Kadener S. circRNA biogenesis competes with pre-mRNA splicing. Mol Cell. 2014;56(1):55-66.

13. Thomson DW, Dinger ME. Endogenous microRNA sponges: evidence and controversy. Nat Rev Genet. 2016;17(5):272-83.

14. Ebbesen KK, Kjems J, Hansen TB. Circular RNAs: identification, biogenesis and function. Biochim Biophys Acta. 2016;1859(1):163-8.

15. Beermann J, Piccoli MT, Viereck J, Thum T. Non-coding RNAs in development and disease: background, mechanisms, and therapeutic approaches. Physiol Rev. 2016;96(4):1297-325.

16. Han B, Chao J, Yao H. Circular RNA and its mechanisms in disease: from the bench to the clinic. Pharmacol Ther. 2018;187:31-44.

17. Meng S, Zhou H, Feng Z, Xu Z, Tang Y, Li P, Wu M. CircRNA: functions and properties of a novel potential biomarker for cancer. Mol Cancer. 2017;16(1):94

18. Wang M, Yu F, Li P. Circular RNAs: Characteristics, Function and Clinical Significance in Hepatocellular Carcinoma. Cancers. 2018;10(8):258.

19. Zhang Y, Liang W, Zhang P, Chen J, Qian H, Zhang X, Xu W. Circular RNAs: emerging cancer biomarkers and targets. J Exp Clin Cancer Res. 2017;36(1):152.

20. Lan X, Xu J, Chen C, Zheng C, Wang J, Cao J, Zhu X, Ge M. The landscape of circular RNA expression profiles in papillary thyroid carcinoma based on RNA sequencing. Cell Physiol Biochem. 2018:47(3):1122-32.

21. Bi W, Huang J, Nie C, Liu B, He G, Han J, Pang R, Ding Z, Xu J, Zhang J. CircRNA circRNA_102171 promotes papillary thyroid cancer progression through modulating CTNNBIP1-dependent activation of beta-catenin pathway. J Exp Clin Cancer Res. 2018;37(1):275

22. Yao $Y$, Chen $X$, Lu S, Zhou C, Xu G, Yan Z, Yang J, Yu T, Chen W, Oian $Y$, et al. Circulating Long noncoding RNAs as biomarkers for predicting head and neck squamous cell carcinoma. Cell Physiol Biochem. 2018; 50(4):1429-40.

23. Sun W, Lan X, Zhang H, Wang Z, Dong W, He L, Zhang T, Zhang P, Liu J, Qin Y. NEAT1_2 functions as a competing endogenous RNA to regulate ATAD2 expression by sponging microRNA-106b-5p in papillary thyroid cancer. Cell Death Dis. 2018;9(3):380.

24. Kim D, Langmead B, Salzberg SL. HISAT: a fast spliced aligner with low memory requirements. Nat Methods. 2015;12(4):357-60.

25. Pertea M, Kim D, Pertea GM, Leek JT, Salzberg SL. Transcript-level expression analysis of RNA-seq experiments with HISAT, StringTie and Ballgown. Nat Protoc. 2016;11(9):1650-67.

26. Uhlen M, Fagerberg L, Hallstrom BM, Lindskog C, Oksvold P, Mardinoglu A, Sivertsson A, Kampf C, Sjostedt E, Asplund A, et al. Proteomics. Tissue-based map of the human proteome. Science. 2015:347(6220):1260419.

27. Griffith OL, Melck A, Jones SJ, Wiseman SM. Meta-analysis and meta-review of thyroid cancer gene expression profiling studies identifies important diagnostic biomarkers. J Clin Oncol. 2006;24(31):5043-51.

28. Wang R, Zhang S, Chen X, Li N, Li J, Jia R, Pan Y, Liang H. CircNT5E acts as a sponge of miR-422a to promote glioblastoma tumorigenesis. Cancer Res. 2018;78(17):4812-25

29. Suzuki H, Zuo Y, Wang J, Zhang MQ, Malhotra A, Mayeda A. Characterization of RNase R-digested cellular RNA source that consists of lariat and circular RNAs from pre-mRNA splicing. Nucleic Acids Res. 2006; 34(8):e63.
30. Stuelten $\mathrm{CH}$, Parent CA, Montell DJ. Cell motility in cancer invasion and metastasis: insights from simple model organisms. Nat Rev Cancer. 2018; 18(5):296-312.

31. Warde-Farley D, Donaldson SL, Comes O, Zuberi K, Badrawi R, Chao P, Franz M, Grouios C, Kazi F, Lopes CT, et al. The GeneMANIA prediction server: biological network integration for gene prioritization and predicting gene function. Nucleic Acids Res. 2010;38(Web Server issue):W214-20.

32. Flores AN, McDermott $N$, Meunier A, Marignol L. NUMB inhibition of $\mathrm{NOTCH}$ signalling as a therapeutic target in prostate cancer. Nat Rev Urol. 2014; 11(9):499-507.

33. Kandachar V, Roegiers F. Endocytosis and control of notch signaling. Curr Opin Cell Biol. 2012;24(4):534-40

34. Dudekula DB, Panda AC, Grammatikakis I, De S, Abdelmohsen K, Gorospe M. Circlnteractome: a web tool for exploring circular RNAs and their interacting proteins and microRNAs. RNA Biol. 2016:13(1):34-42.

35. Li JH, Liu S, Zhou H, Qu LH, Yang JH. starBase v2.0: decoding miRNA-ceRNA, miRNA-ncRNA and protein-RNA interaction networks from large-scale CLIP-Seq data. Nucleic Acids Res. 2014;42(Database issue):D92-7.

36. Yang Y, Ahn YH, Gibbons DL, Zang Y, Lin W, Thilaganathan N, Alvarez CA, Moreira DC, Creighton CJ, Gregory PA, et al. The notch ligand Jagged2 promotes lung adenocarcinoma metastasis through a miR-200-dependent pathway in mice. J Clin Invest. 2011;121(4):1373-85.

37. Fang TC, Yashiro-Ohtani Y, Del Bianco C, Knoblock DM, Blacklow SC, Pear WS. Notch directly regulates Gata3 expression during T helper 2 cell differentiation. Immunity. 2007;27(1):100-10.

38. Malinge S, Thiollier C, Chlon TM, Dore LC, Diebold L, Bluteau O, Mabialah V, Vainchenker W, Dessen P, Winandy S, et al. Ikaros inhibits megakaryopoiesis through functional interaction with GATA-1 and NOTCH signaling. Blood. 2013;121(13):2440-51.

39. Vuong HG, Long NP, Anh NH, Nghi TD, Hieu MV, Hung LP, Nakazawa T, Katoh $\mathrm{R}$, Kondo T. Papillary thyroid carcinoma with tall cell features is as aggressive as tall cell variant: a meta-analysis. Endocr Connect. 2018;7(12): R286-r293.

40. Zanotti S, Canalis E. Notch signaling and the skeleton. Endocr Rev. 2016; 37(3):223-53.

41. Penton AL, Leonard LD, Spinner NB. Notch signaling in human development and disease. Semin Cell Dev Biol. 2012;23(4):450-7.

42. Siebel C, Lendahl U. Notch signaling in development, tissue homeostasis, and disease. Physiol Rev. 2017;97(4):1235-94.

43. Takebe N, Nguyen D, Yang SX. Targeting notch signaling pathway in cancer: clinical development advances and challenges. Pharmacol Ther. 2014;141(2):140-9.

44. Huang Q, Li J, Zheng J, Wei A. The carcinogenic role of the notch signaling pathway in the development of hepatocellular carcinoma. J Cancer. 2019; 10(6):1570-9.

45. Aburjania Z, Jang S, Whitt J, Jaskula-Stzul R, Chen H, Rose JB. The role of Notch3 in Cancer. Oncologist. 2018;23(8):900-11.

46. Aster JC, Pear WS, Blacklow SC. The varied roles of notch in Cancer. Annu Rev Pathol. 2017;12:245-75.

47. Torchy MP, Hamiche A, Klaholz BP. Structure and function insights into the NuRD chromatin remodeling complex. Cell Mol Life Sci. 2015;72(13):2491-507

48. Torrado M, Low JKK, Silva APG, Schmidberger JW, Sana M, Sharifi Tabar M Isilak ME, Winning CS, Kwong C, Bedward MJ, et al. Refinement of the subunit interaction network within the nucleosome remodelling and deacetylase (NuRD) complex. FEBS J. 2017;284(24):4216-32.

49. Wang Z, Kang J, Deng X, Guo B, Wu B, Fan Y. Knockdown of GATAD2A suppresses cell proliferation in thyroid cancer in vitro. Oncol Rep. 2017:37(4):2147-52.

\section{Publisher's Note}

Springer Nature remains neutral with regard to jurisdictional claims in published maps and institutional affiliations. 